Proceedings of the Edinburgh Mathematical Society (2008) 51, 363-385 (C)

DOI:10.1017/S0013091506000034 Printed in the United Kingdom

\title{
TORSION UNITS IN INTEGRAL GROUP RINGS OF CERTAIN METABELIAN GROUPS
}

\author{
MARTIN HERTWECK \\ Universität Stuttgart, Fachbereich Mathematik, IGT, Pfaffenwaldring 5\%, \\ 70569 Stuttgart, Germany (hertweck@mathematik.uni-stuttgart.de)
}

(Received 26 December 2005)

\begin{abstract}
It is shown that any torsion unit of the integral group ring $\mathbb{Z} G$ of a finite group $G$ is rationally conjugate to an element of $\pm G$ if $G=X A$ with $A$ a cyclic normal subgroup of $G$ and $X$ an abelian group (thus confirming a conjecture of Zassenhaus for this particular class of groups, which comprises the class of metacyclic groups).
\end{abstract}

Keywords: integral group ring; torsion unit; bimodule; Zassenhaus conjecture

2000 Mathematics subject classification: Primary 16S34; 16U60

Secondary $20 \mathrm{C} 05$

\section{Introduction}

There is a long-standing conjecture of Zassenhaus that runs as follows.

(ZC1) For a finite group $G$, every torsion unit in $\mathbb{Z} G$ is conjugate to an element of $\pm G$ in the units of $\mathbb{Q} G$.

For known results on the Zassenhaus conjecture $(\mathrm{ZC} 1)$ the reader is referred to $[\mathbf{1 6}$, Chapter 5], [17], $[\mathbf{1 8}, \S 8]$ and $[\mathbf{4}, \mathbf{7}, \mathbf{8}]$.

Certainly the outstanding result in the field is Weiss's proof $[\mathbf{2 0 , 2 1}]$ that the conjecture is true for nilpotent groups $G$. The conjecture has also been verified for some split extensions $G=X \ltimes A$ under coprimeness conditions on $X$ and $A$, with $G$ the metabelian and not too far from being metacyclic: Polcino et al. $[\mathbf{1 5}]$ proved (ZC1) in the case when $X$ and $A$ are cyclic of relatively prime order 20 years ago, but since then no substantial progress has been made beyond this. The main result of this paper is the following theorem.

Theorem 1.1. Suppose that $G=X A$ with $A \unlhd G, X \leqslant G$, and with $A$ cyclic and $X$ abelian. Then (ZC1) holds for $G$.

The proof essentially consists of two parts. In a first step, it is shown that torsion units in $1+\mathrm{I}(\mathbb{Z} A) G$ are rationally conjugate to elements of $A$. (Our notation will be explained in $\S 2$.) The second step consists of showing that, for a torsion unit $u$ not lying 
in $1+\mathrm{I}(\mathbb{Z} A) G$, all partial augmentations $\varepsilon_{a}(u), a \in A$, vanish. Thereby, induction on the order of $G$ and the result from step one is used (see Theorem 7.3 and Corollary 7.5). The main reason for assuming that $A$ is covered by an abelian subgroup $X$, rather than assuming that $G / A$ is abelian, is that these results, once established, are easily shown to hold with $A$ replaced by $\mathrm{C}_{G}(A)$. Then an elementary observation, which we learnt from [4] (see $\S 6$ ), completes the proof.

To establish the first step, we show more precisely that a torsion unit in $1+\mathrm{I}\left(\mathbb{Z} \mathrm{O}_{p}(A)\right) G$ is $p$-adically conjugate to an element of $A$ (see Theorem 5.1 and Claim 5.2). Therefore, we show that, $p$-adically, such a unit has the most simple description one can think of (see Corollary 4.7), which again relies on Weiss's permutation module result [20] as explained in $\S 3$. Then Lemma 2.2, below, allows us to use a result in $[\mathbf{2}]$ to establish the general result (see Corollary 5.3).

Finally, in $\S 8$ we present two results on (ZC1) for direct products of groups which are somehow related to the present work.

Note that [16] may serve as a general reference for any aspects of units in integral group rings. For unexplained concepts of representation theory, we refer the reader to $[\mathbf{3}]$. We believe that the present work can be regarded as 'applied representation theory'.

\section{Review of some known facts}

Throughout this paper, $G$ always denotes a finite group and $R$ will be an integral domain of characteristic zero, with quotient field $K$. For global aspects of torsion units in group rings, we think of $R$ as the coefficient ring $\mathbb{Z}$ or a suitable semi-localization of it, but $R$ may also be a $p$-adic ring, i.e. the integral closure of the $p$-adic integers $\mathbb{Z}_{p}$ in a finite extension field of the $p$-adic field $\mathbb{Q}_{p}$.

Group-theoretical notation is mostly standard. We use the bar convention, set $g^{h}=$ $h^{-1} g h$ and $[g, h]=g^{-1} g^{h}$, let $g^{G}$ be the conjugacy class $\left\{g^{h} \mid h \in G\right\}$ of $g$ in $G$, let ' $\sim$ denote conjugacy and let $\mathrm{O}_{p}(G)$ and $\mathrm{O}_{p^{\prime}}(G)$ denote the largest normal $p$-subgroup and $p^{\prime}$-subgroup of $G$, respectively.

Furthermore, we will adhere to the notation and definitions used in [7]. The most notable notational conventions followed are as follows.

(i) $\varepsilon: R G \rightarrow R$ denotes the augmentation homomorphism.

(ii) $\mathrm{I}(R G)=\{m \in R G \mid \varepsilon(m)=0\}$ denotes the augmentation ideal of $R G$. Thus, for $N \unlhd G, \mathrm{I}(R N) G$ is the kernel of the natural homomorphism $R G \rightarrow R G / N$.

(iii) $\mathrm{V}(R G)$ denotes the group of augmentation 1 units in $R G$; the group of units itself is denoted by $(R G)^{\times}$.

(iv) $\varepsilon_{g}(u)$ denotes the partial augmentation of an element $u$ of $R G$ with respect to the conjugacy class of $g$ in $G$.

(v) ${ }_{1}(R G)_{\alpha}$, for a homomorphism $\alpha$ from a finite group $H$ into $\mathrm{V}(R G)$, denotes the right $R(G \times H)$-module which is $R G$ as an $R$-module, and the group action given by $m \cdot(g, h)=g^{-1} m(h \alpha)$ for all $g \in G, h \in H$ and $m \in R G$. 
The 'bimodules' ${ }_{1}(R G)_{\alpha}$ are the tools used to translate questions about conjugacy into the language of module theory: homomorphisms $\alpha, \beta: H \rightarrow \mathrm{V}(R G)$ are called $R$-equivalent if there exists a unit $v \in(R G)^{\times}$such that $h \alpha=v^{-1} \cdot h \beta \cdot v$ for all $h \in H$; it is easy to see that $\alpha$ and $\beta$ are $R$-equivalent if and only if ${ }_{1}(R G)_{\alpha} \cong{ }_{1}(R G)_{\beta}$ as $R(G \times H)$-modules.

Partial augmentations also make their contribution. A torsion unit $u$ in $\mathrm{V}(R G)$, with no prime divisor on the order of $u$ being invertible in $R$, is conjugate to an element of $G$ in the units of $K G$ if and only if, for every power of $u$, all of its partial augmentations but one vanish (see [13, Theorem 2.5], [16, (41.5)] and [7, Lemma 2.5]). Note that, for $R=\mathbb{Z}$, this is equivalent to saying that all partial augmentations are non-negative.

A connection between both concepts constitutes [21, Lemma 1], also recorded in [16, (38.12)], and is as follows.

Lemma 2.1. For a group homomorphism $\alpha: H \rightarrow \mathrm{V}(R G)$, let $\chi$ be the character of the associated $K(G \times H)$-module $_{1}(K G)_{\alpha}$. Then $\chi((g, h))=\left|\mathrm{C}_{G}(g)\right| \varepsilon_{g}(h \alpha)$ for all $g \in G$, $h \in H$.

The interpretation of partial augmentations as rational multiples of character values of bimodules makes it possible to apply Green's theorem on zeros of characters to obtain (see [7, proof of Theorem 5.6]) the following lemma.

Lemma 2.2. Let $u$ be a torsion unit in $\mathrm{V}(R G)$, where $R$ is a p-adic ring. Suppose that the $p$-part of $u$ is conjugate to an element $x$ of $G$ in the units of $R G$. Then $\varepsilon_{g}(u)=0$ for every $g \in G$ whose $p$-part is not conjugate to $x$.

Proof. We can assume that the $p$-part of $u$ is the group element $x$. Set $M={ }_{1}(R G)_{\iota}$, where $\iota:\langle u\rangle \hookrightarrow \mathrm{V}(R G)$ denotes inclusion, and let $\chi$ be the character of $K M$. The restriction of $M$ to $G \times\langle x\rangle$ is induced from the trivial module $R$ for the diagonal subgroup $\langle(x, x)\rangle$. Thus, $\chi((g, u))=0$ for any $g \in G$ whose $p$-part is not conjugate to $x$ (see $[\mathbf{3}$, (19.27)]). Equivalently, $\varepsilon_{g}(u)=0$ for such $g$, by Lemma 2.1.

This shows that even if one is only interested in rational conjugacy, i.e. in conjugacy which takes place in $(K G)^{\times}$, one may seek $p$-adic conjugacy. The proof of Corollary 5.3 with the help of Theorem 5.1, below, provides a good example of this philosophy; another example is given in [7, Theorem 1.2].

We add some further remarks on partial augmentations of torsion units. The first is pretty elementary but obviously very useful in inductive approaches to the Zassenhaus conjecture (cf. Lemma 6.1 below).

Remark 2.3. Let $u$ be a torsion unit in $\mathrm{V}(R G)$, let $N \unlhd G$ and set $\bar{G}=G / N$. We shall extend the bar convention when writing $\bar{u}$ for the image of $u$ under the natural map $R G \rightarrow R \bar{G}$. Since any conjugacy class of $G$ maps onto a conjugacy class of $\bar{G}$, for any $x \in G$, we have

$$
\varepsilon_{\bar{x}}(\bar{u})=\sum_{g^{G}: \bar{g} \sim \bar{x}} \varepsilon_{g}(u)
$$

In particular, if $N x \subseteq x^{G}$, then $\varepsilon_{\bar{x}}(\bar{u})=\varepsilon_{x}(u)$. 
The next remark shows that, for non-vanishing partial augmentations, there is a nice divisibility property between element orders.

Remark 2.4. Let $u$ be a torsion unit in $\mathrm{V}(\mathbb{Z} G)$. Then $g \in G$ and $\varepsilon_{g}(u) \neq 0$ implies that the order of $g$ divides the order of $u$. Indeed, it is well known that prime divisors on the order of $g$ then divide the order of $u$ (see [13, Theorem 2.7] or [16, (38.11)], as well as [7, Lemma 2.8] for an alternative proof). Furthermore, it was observed in $[\mathbf{8}$, Lemma 5.6] that the orders of the $p$-parts of $g$ cannot exceed those of $u$.

In some sense, there are linear relations between partial augmentations of a torsion unit $u$ and multiplicities of eigenvalues of representing matrices of $u$ which impose constraints on these values (see [12]). Making use of them them is now understood as being the Luthar-Passi method. We shall use this method in the proof of Theorem 7.3 below.

Remark 2.5. Let $u$ be a torsion unit in $\mathrm{V}(\mathbb{Z} G)$. Suppose that $u^{e}=1$ for some natural number $e$ (Zassenhaus [22] has shown that one can always choose $e$ to be the exponent of $G$ ) and assume that $K$ contains a primitive eth root of unity, $\theta$. Let $\chi$ be the character afforded by a $K$-representation $D$ of $G$, and write $\mu(\xi, u, \chi)$ for the multiplicity of an $e$ th root of unity, $\xi$, as an eigenvalue of the matrix $D(u)$. Then (cf. [12], [8, $\S 3]$ )

$$
\mu(\xi, u, \chi)=\frac{1}{e} \sum_{d \mid e} \operatorname{Tr}_{\mathbb{Q}\left(\theta^{d}\right) / \mathbb{Q}}\left(\chi\left(u^{d}\right) \xi^{-d}\right) .
$$

When trying to show that $u$ is rationally conjugate to a group element, one may hope that, by induction on the order of $u$, the values of the summands for $d \neq 1$ are 'known'. The summand for $d=1$ can be written as $(1 / e) \sum_{g^{G}} \varepsilon_{g}(u) \operatorname{Tr}_{\mathbb{Q}(\theta) / \mathbb{Q}}\left(\chi(g) \xi^{-1}\right)$, a linear combination of the $\varepsilon_{g}(u)$ with 'known' coefficients.

\section{Application of Weiss's permutation module result}

Let $N$ be a normal $p$-subgroup of the group $G$, and suppose that a torsion unit $u$ in $\mathrm{V}(R G)$ is given which maps to the identity under the natural map $R G \rightarrow R G / N$. In order to apply Weiss's permutation module result, we assume that $R$ is a $p$-adic ring (with quotient field $K$ ).

It is known that $u$ is of $p$-power order (see $[\mathbf{1 6},(7.5)]$ and $[\mathbf{7}, \S 4]$ ).

Let $\langle c\rangle$ be a cyclic group with 'abstract' generator $c$ of the same order as $u$. Define the homomorphism $\alpha:\langle c\rangle \rightarrow \mathrm{V}(R G)$ by $c \alpha=u$, and write $M^{\alpha}={ }_{1}(R G)_{\alpha}$ for the associated $R(G \times\langle c\rangle)$-module. Then $M^{\alpha}$ is a trivial source module, by Weiss's theorem from $[\mathbf{2 0}],[\mathbf{1 6},(50.1)]$ (cf. [16, (41.12)] and $[\mathbf{7}, \S 4])$.

As a consequence, $u$ is conjugate to an element $x$ of $N$ in the units of $K G$ if $u \in \mathrm{V}(\mathbb{Z} G)$ or, more generally, if $u \in \mathrm{V}\left(\mathbb{Z}_{(G)} G\right)$, where $\mathbb{Z}_{(G)}=\{s / t \mid s, t \in \mathbb{Z},(t,|G|)=1\}$ (see [16, (41.12)] and [7, Proposition 4.2]). We shall assume that this holds (but it is not required for the proof of the next lemma).

Define the homomorphism $\beta:\langle c\rangle \rightarrow \mathrm{V}(R G)$ by $c \beta=x$ and write $M^{\beta}={ }_{1}(R G)_{\beta}$ for the associated $R(G \times\langle c\rangle)$-module. Then $u$ is conjugate to $x$ in the units of $R G$ if and only if $M^{\alpha} \cong M^{\beta}$. By assumption, $K M^{\alpha}$ and $K M^{\beta}$ have the same character. 
Lemma 3.1. The vertices of the indecomposable summands of $M^{\alpha}$ are all contained in $N \times\langle c\rangle$.

Proof. Let $P$ be a Sylow $p$-subgroup of $G$. Then $M^{\alpha}$ is a permutation lattice for $P \times\langle c\rangle$ over $R$, by Weiss's theorem, so

$$
M^{\alpha} \downarrow_{P \times\langle c\rangle} \cong \bigoplus_{i=1}^{n} 1 \uparrow_{U_{i}}^{P \times\langle c\rangle}
$$

for subgroups $U_{1}, \ldots, U_{n}$, and we have to show that $U_{i} \leqslant N \times\langle c\rangle$. The number $n$ of summands equals the $R$-rank of the fixed-point module $\left(M^{\alpha}\right)^{P \times\langle c\rangle}$, so $n=|G: P|$ as $\left(M^{\alpha}\right)^{P \times\langle c\rangle}=\left(M^{\alpha}\right)^{P \times 1}$. Also $\left(M^{\alpha}\right)^{N \times\langle c\rangle}=\left(M^{\alpha}\right)^{N \times 1}$, so the $R$-rank of $\left(M^{\alpha}\right)^{N \times\langle c\rangle}$ is $|G: N|$. For each $i$, Mackey decomposition gives a decomposition

$$
1 \uparrow_{U_{i}}^{P \times\langle c\rangle} \downarrow_{N \times\langle c\rangle}=\bigoplus_{U_{i}(N \times\langle c\rangle) \backslash a} 1 \uparrow_{U_{i}^{a} \cap(N \times\langle c\rangle)}^{N \times\langle c\rangle}
$$

into at most $|P: N|$ summands, and this possible maximal number of summands is only obtained when $U_{i} \leqslant N \times\langle c\rangle$. The resulting estimate for the rank of $\left(M^{\alpha}\right)^{N \times\langle c\rangle}$ proves the assertion.

Using the fact that the restriction of $M^{\alpha}$ to $N \times\langle c\rangle$ is a permutation lattice and that $K M^{\alpha}$ and $K M^{\beta}$ have the same character, it can be shown that the restrictions of $M^{\alpha}$ and $M^{\beta}$ to $N \times\langle c\rangle$ are isomorphic (see [7, Claim 5.1]). Thus, we have the following result.

Corollary 3.2. Under the above assumptions, $M^{\alpha}$ is a direct summand of the direct sum of $|G: N|$ copies of $M^{\beta}$.

Proof. By Lemma 3.1, $M^{\alpha}$ is relatively projective to $R(N \times\langle c\rangle)$, so that $M^{\alpha}$ is a direct summand of $M_{N \times\langle c\rangle}^{\alpha} \uparrow G \times\langle c\rangle$ (see [3, (19.5)]). Since $M_{N \times\langle c\rangle}^{\beta} \uparrow G \times\langle c\rangle$ is clearly isomorphic to the direct sum of $|G: N|$ copies of $M^{\beta}$, the claim follows from the remark preceding the corollary.

The following corollary is a simple prototype for intended applications, and will be used in $\S 8$ to give another proof that the Zassenhaus conjecture (ZC1) holds for nilpotent groups. Another application to $p$-adic conjugacy of torsion units will be given in Theorem 5.1 with the help of Lemma 4.6.

Corollary 3.3. Let $N$ be a $p$-group and let $H$ be a $p^{\prime}$-group. Then every torsion unit of p-power order in $\mathrm{V}(\mathbb{Z}(H \times N))$ is conjugate to an element of $N$ in the units of $\mathbb{Z}_{p}(H \times N)$.

Proof. Set $G=H \times N$ and $R=\mathbb{Z}_{p}$, and let $u$ be a torsion unit of $p$-power order in $\mathrm{V}(\mathbb{Z} G)$. Then $u$ maps to 1 under the natural map $\mathbb{Z} G \rightarrow \mathbb{Z} G / N=\mathbb{Z} H$ (see [16, (1.9)]).

As already remarked, $u$ is conjugate to an element $x$ of $N$ in the units of $K G$; let $M^{\alpha}$ and $M^{\beta}$ be the bimodules defined above. We show that $M^{\alpha} \cong M^{\beta}$. 
Let $1=e_{1}+\cdots+e_{s}$ be an orthogonal decomposition into primitive idempotents of $R H$. Then $R G=R G e_{1} \oplus \cdots \oplus R G e_{s}$ is a decomposition into indecomposable (projective) $R G$-modules which at the same time is a decomposition of $R G$ as an $(R G, R N)$-bimodule. We remark that two summands $R G e_{i}$ and $R G e_{j}$ are isomorphic as $(R G, R N)$-bimodules provided they are isomorphic as $R G$-modules: Indeed, $R G e_{i \downarrow} \downarrow_{H}$ is a direct sum of copies of the indecomposable module $R H e_{i}$, so $R G e_{i} \cong R G e_{j}$ as $R G$-modules implies that $R H e_{i} \cong R H e_{j}$ as $R H$-modules, i.e. $e_{j}=e_{i}^{v}$ for some $v \in(R H)^{\times}$, and $R G e_{i} \stackrel{\cdot v}{\longrightarrow} R G e_{j}$ is an isomorphism of $(R G, R N)$-bimodules.

We have noted that $M^{\beta}=R G e_{1} \oplus \cdots \oplus R G e_{s}$ is a decomposition into indecomposables and, by Corollary 3.2, each indecomposable summand of $M^{\alpha}$ is among the $R G e_{i}$. Since $M^{\alpha} \cong M^{\beta}$ as $R G$-modules, the claim now follows from the remark made in the previous paragraph.

\section{Indecomposable summands of some bimodules}

We note some elementary facts about indecomposable summands of bimodules such as $M^{\alpha}$ and $M^{\beta}$ from the last section.

Remark 4.1. For the moment, $R$ may denote any commutative ring. Let $U$ be a finite subgroup of $\mathrm{V}(R G)$. We write $(R G)^{U}=\left\{m \in R G \mid m^{u}=m\right.$ for all $\left.u \in U\right\}$ for the subring of $U$-invariants of $R G$.

Let $\iota: U \hookrightarrow \mathrm{V}(R G)$ denote inclusion. Decompositions ${ }_{1}(R G)_{\iota}=M_{1} \oplus \cdots \oplus M_{s}$ of the $R(G \times U)$-module ${ }_{1}(R G)_{\iota}$ correspond to orthogonal idempotent decompositions $1=e_{1}+\cdots+e_{s}$ in $(R G)^{U}$, the correspondence given by $M_{i}=R G e_{i}$. The summand $M_{i}$ is indecomposable if and only if $e_{i}$ is primitive in $(R G)^{U}$.

For an idempotent $e$ in $(R G)^{U}$, the isomorphism $\operatorname{End}_{R G}(R G e) \cong e R G e$ (given by $\varphi \mapsto e \varphi)$ restricts to an isomorphism $\operatorname{End}_{R(G \times U)}(R G e) \cong e(R G)^{U} e$.

Suppose that $R G e \cong R G f$ as $R(G \times U)$-modules for some idempotents $e$ and $f$ in $(R G)^{U}$. Clearly, $R G e \oplus R G(1-e) \cong R G \cong R G f \oplus R G(1-f)$. If the Krull-Schmidt theorem holds, then $R G(1-e) \cong R G(1-f)$ by cancellation, whence the isomorphism $R G e \cong R G f$ is given by right multiplication with a unit $v$ in $(R G)^{U}$ and $f=e^{v}$ (cf. $[3, \S 6$, Exercises 14, 15]).

In all cases we are looking at, the Krull-Schmidt theorem is assumed to hold.

We may have given $U$ as an abstract group and wish to 'compare' different (injective) homomorphisms from $U$ into $\mathrm{V}(R G)$ via the associated bimodules. Then similar remarks hold. We only note the following.

Remark 4.2. Let $M^{\alpha}$ and $M^{\beta}$ be as in $\S 3$. Let $e$ be an idempotent in $(R G)^{\langle u\rangle}$, let $f$ be an idempotent in $(R G)^{\langle x\rangle}$, and consider $R G e$ and $R G f$ as submodules of $M^{\alpha}$ and $M^{\beta}$, respectively. Then any isomorphism $R G e \cong R G f$ is given by multiplication with a unit $v$ of $R G$ such that $f=e^{v}$ and $f u^{v}=u^{v} f=x f$.

Primitive idempotent decompositions which take place in $R L$, for some normal subgroup $L$ of $G$, may prove useful in analysing the bimodules. We note the following obvious instance. 
Claim 4.3. Let $L \unlhd G$ and let $\mu$ and $\nu$ be primitive idempotents in $R L$. Suppose that the $R G$-modules $R G \mu$ and $R G \nu$ have a common (non-zero) direct summand. Then $R G \mu \cong R G \nu$. More precisely, $\nu=\mu^{g v}$ for some $g \in G$ and $v \in(R L)^{\times}$.

Suppose further that $L \leqslant \mathrm{C}_{G}(H)$ for a subgroup $H$ of $G$, and let $\iota: H \hookrightarrow \mathrm{V}(R G)$ denote inclusion. Then $R G \nu$ and $R G \mu^{g}$ are isomorphic direct summands of the $R(G \times H)$ module ${ }_{1}(R G)_{\iota}$ (with an isomorphism given by right multiplication with $v^{-1}$ ).

Proof. Let $G=\bigcup_{i} L g_{i}$ be the disjoint union of the cosets of $L$. Then $R G \mu=R L \mu \uparrow^{G}=$ $\bigoplus_{i} g_{i}^{-1} R L \mu$ as $R L$-modules, and $g^{-1} R L \mu \stackrel{\cdot g}{\rightarrow} R L \mu^{g}$ is an isomorphism of $R L$-modules for any $g \in G$, so $R G \mu_{\downarrow} \cong \bigoplus_{i} R L \mu^{g_{i}}$ is a direct sum of indecomposable $R L$-modules (the $\mu^{g_{i}}$ are primitive in $R L$ since $\left.L \unlhd G\right)$. Likewise, $R G \nu_{\downarrow} \cong \bigoplus_{i} R L \nu^{g_{i}}$, and since $R G \mu \downarrow_{L}$ and $R G \nu_{\downarrow}$ have a common direct summand, we have $R L \nu^{g_{i}} \cong R L \mu^{g_{j}}$ for some indices $i, j$, that is, $\nu^{g_{i}}$ and $\mu^{g_{j}}$ are conjugate by a unit in $R L$.

The additional statement concerning the bimodule is obvious.

The normal subgroups we are interested in are the centralizers of normal $p$-subgroups of $G$, as will become clear from the following discussion.

Remark 4.4. Let $N$ be a normal $p$-subgroup of $G$ and set $L=\mathrm{C}_{G}(N)$. (Then $L \unlhd G$, but this does not matter for the moment.) Let $R$ be a commutative local ring whose residue class field has characteristic $p$. Set

$$
J=\left\langle\sum_{h \in g^{N}} h \mid g \in G \backslash L\right\rangle_{(R G)^{N}},
$$

the two-sided ideal of $(R G)^{N}$ generated by all $N$-conjugacy class sums in $G$ of length larger than 1. We have $(R G)^{N}=R L+J$. Since, for $g \in G \backslash L$,

$$
\sum_{h \in g^{N}} h \in\left|g^{N}\right| g+\mathrm{I}(R N) G \subseteq \mathrm{I}(R N) G+p R G,
$$

$J$ is nilpotent modulo $p(R G)^{N}$ and hence $J \subseteq \operatorname{rad}(R G)^{N}$ (see [3, (5.26)]). Thus, the inclusion $R L \hookrightarrow(R G)^{N}$ is a covering homomorphism (in the sense of $[\mathbf{1 9}, \S 25]$ ), i.e. $(R G)^{N}=R L+\operatorname{rad}(R G)^{N}$.

Let $e$ be an idempotent in $R L$. Since $\operatorname{rad} e(R G)^{N} e=e\left(\operatorname{rad}(R G)^{N}\right) e(\operatorname{see}[\mathbf{3},(5.13)])$, $e J e$ is contained in the radical of $e(R G)^{N} e$, and $e R L e \hookrightarrow e(R G)^{N} e$ is also a covering homomorphism. Suppose that $e$ is primitive in $R L$. Then $e R L e$ is local (since $e R L e$ has no non-trivial idempotents and the radical quotient of $e R L e$ is semisimple artinean $[\mathbf{3}$, $(5.22)])$, so $e R L e \hookrightarrow e(R G)^{N} e$ induces an isomorphism on the radical quotients, and $e(R G)^{N} e$ is local.

We explicitly record what we are interested in. Let $N$ be a normal $p$-subgroup of $G$ and let $R$ be a $p$-adic ring.

Remark 4.5. Let $e$ be a primitive idempotent in $R \mathrm{C}_{G}(N)$. Then $e(R G)^{N} e$ is a local ring, and hence $R G e$ is an indecomposable $R(G \times N)$-module. 
In combination with Claim 4.3 we obtain the following lemma.

Lemma 4.6. Let $\varphi: U \rightarrow N$ be a group isomorphism and set $\alpha: U \stackrel{\varphi}{\rightarrow} N \hookrightarrow \mathrm{V}(R G)$. Suppose that $M$ is an $R(G \times U)$-module which is free of rank 1 as an $R G$-module and a direct summand of a direct sum of copies of ${ }_{1}(R G)_{\alpha}$. Let $1=e_{1}+\cdots+e_{s}$ be an orthogonal decomposition into primitive idempotents of $R \mathrm{C}_{G}(N)$. Then there exist $g_{1}, \ldots, g_{s} \in G$ such that $M \cong R G e_{1}^{g_{1}} \oplus \cdots \oplus R G e_{s}^{g_{s}}$, where each summand $R G e_{i}^{g_{i}}$ is considered as a submodule of ${ }_{1}(R G)_{\alpha}$. In other words, $M \cong{ }_{1}(R G)_{\gamma}$, where $\gamma$ is the homomorphism $U \rightarrow \mathrm{V}(R G)$ given by $u \mapsto \sum_{i} e_{i}(u \varphi)^{g_{i}^{-1}}$ for $u \in U$.

Proof. We have ${ }_{1}(R G)_{\iota}=R G e_{1} \oplus \cdots \oplus R G e_{s}$, where each summand is an indecomposable $R(G \times U)$-module by Remark 4.5. By assumption, the Krull-Schmidt theorem provides an isomorphism $M \cong R G f_{1} \oplus \cdots \oplus R G f_{t}$, where each $f_{i}$ is taken from the set $\left\{e_{1}, \ldots, e_{s}\right\}$ (some of them may be equal). Thus, as $R G$-modules,

$$
R G e_{1} \oplus \cdots \oplus R G e_{s}=R G \cong R G f_{1} \oplus \cdots \oplus R G f_{t} .
$$

Renumbering if necessary, we can assume that $R G e_{1}$ and $R G f_{1}$ as $R G$-modules have a direct summand in common. Then $f_{1}=e_{1}^{g_{1}}$ for some $g_{1} \in G$, by Claim 4.3. Cancelling the isomorphic summands $R G e_{1}$ and $R G f_{1}$ in (4.1) and continuing this way proves the lemma.

Corollary 4.7. Suppose that $N$ is cyclic. Let $u$ be a torsion unit in $1+\mathrm{I}(\mathbb{Z} N) G$. Then $u$ is conjugate to an element $x$ of $N$ in the units of $\mathbb{Q} G$, and we can assume that $N=\langle x\rangle$. If $1=e_{1}+\cdots+e_{s}$ is an orthogonal decomposition into primitive idempotents of $R \mathrm{C}_{G}(x)$, then there exist $g_{1}, \ldots, g_{s} \in G$ such that $u$ is conjugate in $(R G)^{\times}$to $\sum_{i=1}^{s} e_{i} x^{g_{i}}$.

Proof. This is immediate from the above discussion (see Corollary 3.2 and Lemma 4.6).

Even if $N$ is not cyclic, the above facts may be useful for obtaining information about torsion units in $1+\mathrm{I}(\mathbb{Z} N) G$, since a statement corresponding to Remark 4.5 can be made for $p$-elements in $N$ : if $x \in \mathrm{O}_{p}(G)$ and $e$ is a primitive idempotent in $R \mathrm{C}_{G}(x)$, then $e(R G)^{\langle x\rangle} e$ is a local ring. This may be particularly promising for metabelian groups when $\mathrm{C}_{G}(x)$ is likely to be a normal subgroup of $G$.

Remark 4.8. Let $x \in \mathrm{O}_{p}(G)$. Then $N=\left\langle x^{g} \mid g \in G\right\rangle$ is a normal $p$-subgroup of $G$. Suppose that $\left[G, \mathrm{C}_{G}(x)\right] \leqslant \mathrm{C}_{G}(N)$. Then $\mathrm{C}_{G}(x)=\mathrm{C}_{G}(N)$. Indeed, for $g, h \in G$ with $h \in \mathrm{C}_{G}(x)$, we have $x^{g h}=x^{g[g, h]}=x^{g}$, showing that $h \in \mathrm{C}_{G}(N)$. For example, if $G$ is metabelian and $x \in \mathrm{O}_{p}\left(G^{\prime}\right)$, then $\mathrm{C}_{G}(x)=\mathrm{C}_{G}(N)$.

\section{Torsion units in $1+\mathrm{I}(\mathbb{Z} A) G$}

The aim of this section is to present the following consequence of Corollary 4.7. It will be applied to torsion units in $1+\mathrm{I}(\mathbb{Z} A) G$, for $G$ and $A$ as in Theorem 1.1. 
Theorem 5.1. Suppose that $N$ is a cyclic normal p-subgroup of $G$. Let $u$ be a torsion unit in $1+\mathrm{I}(\mathbb{Z} N) G$. Then $u$ is conjugate to an element $x$ of $N$ in the units of $\mathbb{Q} G$. If $\mathrm{C}_{G}(x)$ has a normal $p$-complement, then $u$ is even $p$-adically conjugate to $x$.

Proof. We have already noted in $\S 3$ that $u$ is conjugate to an element $x$ of $N$ in the units of $\mathbb{Q} G$. We can assume that $N=\langle x\rangle$, and set $L=\mathrm{C}_{G}(N) \unlhd G$. By assumption, $L / \mathrm{O}_{p^{\prime}}(L)$ is a $p$-group.

Let $R$ be a $p$-adic ring such that its field of quotients $K$ is sufficiently large for $G$. We will show that $u$ and $x$ are conjugate in the units of $R G$. Interpreting the units as bimodules and using a result due to Reiner and Zassenhaus [3, (30.25)], it then follows that conjugacy already takes place in the units of $\mathbb{Z}_{p} G$.

The group $L$ acts on the centrally primitive idempotents of $R \mathrm{O}_{p^{\prime}}(L)$ and, since $L \unlhd G$, the group $G$ acts on the orbits of this $L$-action. Let $e$ be the sum of the centrally primitive idempotents of $R \mathrm{O}_{p^{\prime}}(L)$ which lie in an orbit under the $L$-action. Set $T=\{g \in G \mid$ $\left.e^{g}=e\right\}$, the inertia group of $e$ in $G$. Note that $L \leqslant T \unlhd G$, since $G / L$ can be identified with a subgroup of the abelian group $\operatorname{Aut}(N)$. Set $n=|G: T|$, and let $s_{1}, \ldots, s_{n}$ be representatives of the cosets of $T$ in $G$. Let $\epsilon$ be the sum of the $G$-conjugates of $e$, so that $\epsilon=\sum_{j=1}^{n} e^{s_{j}^{-1}}$, a central idempotent of $R G$.

We remark that a primitive idempotent in $R \mathrm{O}_{p^{\prime}}(L)$ remains primitive in $R L$ since $L / \mathrm{O}_{p^{\prime}}(L)$ is a $p$-group, by Green's indecomposability theorem (see $[\mathbf{3},(19.23)]$ or $[\mathbf{1 9}$, $\S 23]$ ). Write $e=e_{1}+\cdots+e_{m}$ with orthogonal primitive idempotents in $R \mathrm{O}_{p^{\prime}}(L)$. Then

$$
\epsilon=\sum_{i=1}^{m} \sum_{j=1}^{n} e_{i}^{s_{j}^{-1}}
$$

is a decomposition into orthogonal primitive idempotents of $R L$.

By Corollary 4.7, there exist $g_{i j} \in G(1 \leqslant i \leqslant m, 1 \leqslant j \leqslant n)$ such that $\epsilon u$ is conjugate in $(\epsilon R G)^{\times}$to the element

$$
v=\sum_{i=1}^{m} \sum_{j=1}^{n} e_{i}^{s_{j}^{-1}} x^{g_{i j}} .
$$

We know that $v$ is conjugate to $\epsilon x$ in $(\epsilon K G)^{\times}$, and have to show that conjugacy already takes place in $(\epsilon R G)^{\times}$. To this end, we will compare the eigenvalues of $v$ and $\epsilon x$ with reference to a suitably chosen representation.

$\epsilon R G \cong \operatorname{Mat}_{n}(e R T e)$ is a well-known result due to Clifford (see [14, $\S 6$, Lemma 1.7] or [6, Theorem 2.5]). The isomorphism can be chosen such that the elements $\epsilon x$ and $v$ of $R T$ (remember that $T \unlhd G$ ) correspond to diagonal matrices:

$$
\begin{aligned}
\epsilon x & \mapsto \operatorname{diag}\left(e x^{s_{1}}, \ldots, e x^{s_{n}}\right), \\
v & \mapsto \operatorname{diag}\left(\sum_{i=1}^{m} e_{i} x^{g_{i 1} s_{1}}, \ldots, \sum_{i=1}^{m} e_{i} x^{g_{i n} s_{n}}\right) .
\end{aligned}
$$

Let $\psi$ be the sum of the irreducible characters of $K \mathrm{O}_{p^{\prime}}(L)$ which belong to $e K \mathrm{O}_{p^{\prime}}(L)$. By our assumption on $R$, we have $\psi(f)=1$ for every primitive idempotent $f$ of $R \mathrm{O}_{p^{\prime}}(L)$ 
satisfying ef $\neq 0$, so, in particular, $\psi(1)=m$. Let $\pi: N \rightarrow K$ be a faithful character and set $\xi=\pi(x)$, so $\xi$ is a primitive $|N|$ th root of unity. The tensor product $\psi \otimes \pi$ is a character of $\mathrm{O}_{p^{\prime}}(L) \times N$ and we set $\chi=(\psi \otimes \pi) \uparrow^{T}$. Let $D$ be a representation affording $\chi$. Set $q=\left|L: \mathrm{O}_{p^{\prime}}(L) \times N\right|$, and let $t_{1}, \ldots, t_{r}$ be representatives of the cosets of $L$ in $T$ (so $r=|T: L|$ ). For representatives $s_{j}, t_{k}$, let $\sigma_{j}, \tau_{k} \in \operatorname{Gal}(\mathbb{Q}(\xi) / \mathbb{Q})$ be defined by $\pi\left(x^{s_{j}}\right)=\xi^{\sigma_{j}}$ and $\pi\left(x^{t_{k}}\right)=\xi^{\tau_{k}}$. Also, let $\gamma_{i j} \in \operatorname{Gal}(\mathbb{Q}(\xi) / \mathbb{Q})$ be defined by $\pi\left(x^{g_{i j}}\right)=\xi^{\gamma_{i j}}$.

Now suppose that we are given $g_{1}, \ldots, g_{m} \in G$ and that the $\gamma_{i} \in \operatorname{Gal}(\mathbb{Q}(\xi) / \mathbb{Q})$ are defined by $\pi\left(x^{g_{i}}\right)=\xi^{\gamma_{i}}$. Then $(\psi \otimes \pi) \uparrow^{L}\left(e_{i} x^{g_{i}}\right)=q \xi^{\gamma_{i}}, \chi\left(e_{i} x^{g_{i}}\right)=q \sum_{k=1}^{r} \xi^{\tau_{k} \gamma_{i}}$ and

$$
\chi\left(\sum_{i=1}^{m} e_{i} x^{g_{i}}\right)=q \sum_{i=1}^{m} \sum_{k=1}^{r} \xi^{\tau_{k} \gamma_{i}} .
$$

However, if we examine the affording representations, we see more precisely that the $\xi^{\tau_{k}} \gamma_{i}$ are the eigenvalues of the representing matrix $D\left(\sum_{i=1}^{m} e_{i} x^{g_{i}}\right)$, each one occurring $q$ times the number of times it occurs in the list $\xi^{\tau_{1} \gamma_{1}}, \xi^{\tau_{1} \gamma_{2}}, \ldots$

Let $\hat{D}$ be the representation of $K G$ obtained by 'composing' the homomorphism $K G \rightarrow$ $\epsilon K G \cong \operatorname{Mat}_{n}(e K T e)$ with the representation $D$ of $K T$, and let $\hat{\chi}$ be its character. Then

$$
\hat{\chi}(\epsilon x)=q m \sum_{j=1}^{n} \sum_{k=1}^{r} \xi^{\tau_{k} \sigma_{j}}, \quad \hat{\chi}(v)=q \sum_{i=1}^{m} \sum_{j=1}^{n} \sum_{k=1}^{r} \xi^{\tau_{k} \gamma_{i j} \sigma_{j}},
$$

where the occurring roots of unity are the eigenvalues (including multiplicities) of the representing matrices $\hat{D}(\epsilon x)$ and $\hat{D}(v)$. They agree for both matrices, since $\epsilon x$ and $v$ are rationally conjugate, and so:

for every $1 \leqslant j_{0} \leqslant n$, there exist exactly $m$ pairs $(i, j)$ such that

$$
g_{i j} s_{j} \equiv s_{j_{0}} \bmod T \text {, which will be indicated by }(i, j) \rightsquigarrow j_{0} .
$$

This is all the information we need, so we now turn to the bimodules.

Let $M^{\alpha}$ and $M^{\beta}$ be defined as in $\S 3$, so we have a generator $c$ of an 'abstract' cyclic group $\langle c\rangle$ of the same order as $u$, and $M^{\alpha}$ and $M^{\beta}$ are the right $R(G \times\langle c\rangle)$-modules, which are simply $R G$ with $R G$ acting from the left and $c$ acting by right multiplication with $u$ and $x$, respectively.

We have to show that the direct summands $\epsilon M^{\alpha}$ and $\epsilon M^{\beta}$ are isomorphic, and therefore we will change the action of $\langle c\rangle$ on $\epsilon M^{\alpha}$ by letting $c$ act by multiplication with $v$ (this does not change the isomorphism type). Note that

$$
\epsilon R G=\bigoplus_{i, j} R G e_{i}^{s_{j}^{-1}}
$$

is a direct sum decomposition of both $\epsilon M^{\alpha}$ and $\epsilon M^{\beta}$. We shall write $\epsilon M_{i j}^{\alpha}$ and $\epsilon M_{i j}^{\beta}$ for $R G e_{i}^{s_{j}}$ when considered as direct summand of $\epsilon M^{\alpha}$ and $\epsilon M^{\beta}$, respectively.

Fix some $1 \leqslant j_{0} \leqslant n$. Note that $\epsilon M_{i_{1} j_{0}}^{\beta} \cong \epsilon M_{i_{2} j_{0}}^{\beta}$ for all $1 \leqslant i_{1}, i_{2} \leqslant m$. This is simply because $e_{i_{1}}$ and $e_{i_{2}}$ are conjugate by a unit in $R L$ and $L=\mathrm{C}_{G}(x) \unlhd G$. Also

$$
M^{\beta} e_{i}^{s_{j_{0}}^{-1}} \cong M^{\beta} e_{i}^{t s_{j_{0}}^{-1}} \text { for all } 1 \leqslant i \leqslant m \text { and } t \in T,
$$


again since $e_{i}$ and $e_{i}^{t}$ are conjugate by a unit in $R L$. Now if $(i, j) \rightsquigarrow j_{0}$, then

$$
\epsilon M_{i j_{0}}^{\beta}=M^{\beta} e_{i}^{s_{j_{0}}^{-1}} \cong M^{\beta} e_{i}^{s_{j}^{-1} g_{i j}^{-1}}
$$

and multiplication with $g_{i j}$ gives an isomorphism

$$
M^{\beta} e_{i}^{s_{j}^{-1} g_{i j}^{-1}} \cong \epsilon M_{i j}^{\alpha},
$$

so $\epsilon M_{i j_{0}}^{\beta} \cong \epsilon M_{i j}^{\alpha}$. Thus, it follows from (5.1) that

$$
\bigoplus_{(i, j) \rightsquigarrow j_{0}} \epsilon M_{i j}^{\alpha} \cong \bigoplus_{(i, j) \rightsquigarrow j_{0}} \epsilon M_{i j_{0}}^{\beta} \cong \bigoplus_{i=1}^{m} \epsilon M_{i j_{0}}^{\beta}
$$

and

$$
\epsilon M^{\alpha}=\bigoplus_{j_{0}=1}^{n}\left(\bigoplus_{(i, j) \rightsquigarrow j_{0}} \epsilon M_{i j}^{\alpha}\right) \cong \bigoplus_{j_{0}=1}^{n}\left(\bigoplus_{i=1}^{m} \epsilon M_{i j_{0}}^{\beta}\right)=\epsilon M^{\beta}
$$

The proof is complete.

The assumption on $\mathrm{C}_{G}(x)$ in Theorem 5.1 has been chosen so that it applies to the groups of our favourite class, as follows.

Claim 5.2. Suppose that $G=X A$ for $A \unlhd G, X \leqslant G$ with $A$ cyclic, $X$ abelian and $A$ having a non-trivial p-subgroup $N$. Then $\mathrm{C}_{G}(N)$ has a normal p-complement.

Proof. Set $\bar{G}=G / \mathrm{O}_{p^{\prime}}(G)$. From $\mathrm{O}_{p^{\prime}}(G) \leqslant \mathrm{C}_{G}(N)$ and $\mathrm{O}_{p^{\prime}}(G) \cap N=1$ it follows that $\mathrm{C}_{G}(N)$ is the pre-image of $\mathrm{C}_{\bar{G}}(\bar{N})$ under the natural map $G \rightarrow \bar{G}$. We proceed to show that $\mathrm{C}_{\bar{G}}(\bar{N})$ is a $p$-group. Let $x$ be a $p^{\prime}$-element of $X$. If $[\bar{x}, \bar{A}]=1$, then $\bar{x} \in \mathrm{Z}(G)$ and $\bar{x} \in \mathrm{O}_{p^{\prime}}(\bar{G})$. Otherwise, $\bar{x}$ acts fixed-point freely on the cyclic $p$-group $\bar{A}$ and $[\bar{x}, \bar{N}] \neq 1$. Since $A \leqslant \mathrm{C}_{G}(N)$, this gives the desired results, i.e. $\mathrm{O}_{p^{\prime}}(G)$ is the normal $p$-complement of $\mathrm{C}_{G}(N)$.

Now we can use a theorem of Cliff and Weiss [2, Theorem 6.3] to deduce the following result.

Corollary 5.3. Suppose that $G=X A$ with $A \unlhd G, X \leqslant G$ and with $A$ cyclic and $X$ abelian. Then any torsion unit in $1+\mathrm{I}\left(\mathbb{Z}_{G}(A)\right) G$ is rationally conjugate to an element of $G$.

Proof. First, let $u$ be a torsion unit in $1+\mathrm{I}(\mathbb{Z} A) G$. If $u$ is a $p$-element, for some prime $p$, then $u \in 1+\mathrm{I}\left(\mathbb{Z} \mathrm{O}_{p}(A)\right) G$ (see $\left.[\mathbf{1 6},(7.5)]\right)$, so $u$ is $p$-adically conjugate to an element of $A$ by Theorem 5.1 and Claim 5.2. Thus, for $u$ of arbitrary order, $\varepsilon_{g}(u)=0$ for all $g \in G \backslash A$ by Lemma 2.2 and Remark 2.4. For $m=|G: A|$, we have an embedding of $R G$ into $(R A)_{m}$, the ring of $m \times m$ matrices over $R A$. Let $U$ be the image of $u$ under this embedding. Since $U$ lies in the kernel $\operatorname{SGL}(R A)$ of the augmentation map $\mathrm{GL}_{m}(R A) \rightarrow \mathrm{GL}_{m}(R)$ and $A$ is cyclic, it follows from [2, Theorem 6.3] that $\varepsilon_{a}(u) \geqslant 0$ 
for all $a \in A$ (note the well-known relation (7.1)). Thus, $\varepsilon_{g}(u) \geqslant 0$ for all $g \in G$, and since the same holds for all powers of $u$, it follows that $u$ is rationally conjugate to an element of $G$ (see $[\mathbf{1 6},(41.5)])$.

Now let $u$ be a torsion unit in $1+\mathrm{I}\left(\mathbb{Z}_{G}(A)\right) G$. Set $\bar{G}=G / A$. Then $\bar{u}=\bar{g}$ in $\mathbb{Z} \bar{G}$ for some $g \in \mathrm{C}_{G}(A)=A \mathrm{C}_{X}(A)$, since $\bar{G}$ is abelian and $\bar{u}$ maps to the identity in $\mathbb{Z} \bar{G} / \overline{\mathrm{C}_{G}(A)}$. We can assume that $g \in \mathrm{C}_{X}(A) \subseteq \mathrm{Z}(G)$, when $g^{-1} u$ is a torsion unit in $1+\mathrm{I}(\mathbb{Z} A) G$. Thus, $u$ is rationally conjugate to an element of $G$ by the preceding result.

\section{An inductive argument for metabelian groups}

The following simple group-theoretical observation is taken from [4, Lemma 2]. It immediately yields information about partial augmentations of torsion units in integral group rings of metabelian groups if one takes an inductive approach (cf. Remark 2.3).

Lemma 6.1. Let $A$ be an abelian normal subgroup of $G$, and suppose $g \in G$ satisfies $[g, G] \leqslant A$. Then $B=[g, A] \unlhd G$ and $B g^{G}=g^{G}$. (That is, $g^{G}$ is the only conjugacy class of $G$ which maps onto the conjugacy class of $B g$ in $G / B$.)

Proof. Since $A$ is abelian, elementary commutator calculus shows that $B \unlhd G$. Let $a \in A$ and $h \in G$. Then

$$
\begin{aligned}
{[g, a] g^{h} } & =g^{-1} a^{-1} g \cdot a \cdot h^{-1} g h g^{-1} \cdot g \\
& =g^{-1} a^{-1} g \cdot h^{-1} g h g^{-1} \cdot a \cdot g \quad(\text { since } A \text { is abelian) } \\
& =g^{h g^{-1} a g} \in g^{G} .
\end{aligned}
$$

Since each element of $B$ can be written as a product of elements of the form $[g, a], a \in A$, the statement of the lemma follows.

Corollary 6.2. Suppose that $G$ has an abelian normal subgroup $A$ with abelian quotient $G / A$, and that the Zassenhaus conjecture (ZC1) holds for proper quotients of $G$. Let $u$ be a torsion unit in $\mathrm{V}(\mathbb{Z} G)$. Then the following hold.

(i) $\varepsilon_{g}(u) \in\{0,1\}$ for all $g \in G \backslash \mathrm{C}_{G}(A)$.

(ii) Set $G^{*}=G / \mathrm{C}_{G}(A)$ and suppose that $u^{*} \neq 1$. Then there exists (up to conjugacy) a unique $g_{0} \in G \backslash \mathrm{C}_{G}(A)$ with $\varepsilon_{g_{0}}(u)=1$, and $\varepsilon_{x}(u)=0$ for all $x \in G \backslash \mathrm{C}_{G}(A)$ with $x \notin g_{0}^{G}$ (obviously $u^{*}=g_{0}^{*}$ ).

(iii) Let $u$ and $g_{0}$ be as in (ii). Let $N$ be a non-trivial normal subgroup of $G$ contained in $\mathrm{C}_{G}(A)$ and set $\bar{G}=G / N$. Then $\bar{u}$ is conjugate to $\bar{g}_{0}$ in $(\mathbb{Q} \bar{G})^{\times}$.

Proof. (i) Let $g \in G \backslash \mathrm{C}_{G}(A)$ and set $B=[g, A]$. Then $1 \neq B \unlhd G$ and (ZC1) holds for $\bar{G}=G / B$. Therefore, all but one of the partial augmentations of $\bar{u}$ vanish and since, by Lemma $6.1, g^{G}$ is the unique conjugacy class of $G$ which maps onto $\bar{g}^{\bar{G}}$ in $\bar{G}$, it follows that $\varepsilon_{g}(u)=\varepsilon_{\bar{g}}(\bar{u}) \in\{0,1\}$. 
(ii) By a well-known result of Berman and Higman, $u^{*}$ has zero 1-coefficient (see [16, (1.4)]), and since $u^{*}$ has augmentation 1, the claim follows from (i) and Remark 2.3.

(iii) We have $\varepsilon_{\bar{g}_{0}}(\bar{u})=\sum_{g^{G}: \bar{g} \sim \bar{g}_{0}} \varepsilon_{g}(u)$, and since $G / \mathrm{C}_{G}(A)$ is abelian, the sum extends over certain classes $g^{G}$ with $g \in g_{0} \mathrm{C}_{G}(A)$, so $\varepsilon_{\bar{g}_{0}}(\bar{u})=\varepsilon_{g_{0}}(u)=1$ by (ii). Since $(\mathrm{ZC} 1)$ holds for $\bar{G}$, the claim follows.

Remark 6.3. Let $u$ be a torsion unit in $\mathrm{V}(\mathbb{Z} G)$. Let $N$ be a normal $p$-subgroup of $G$, set $\bar{G}=G / N$ and assume that $\bar{u} \sim \bar{g}$ in $(\mathbb{Q} \bar{G})^{\times}$for some $g \in G$. Then the $p^{\prime}$-parts of $u$ and $g$ have the same order. This holds since torsion units in the kernel of $\mathrm{V}(\mathbb{Z} G) \rightarrow \mathrm{V}(\mathbb{Z} \bar{G})$ have $p$-power order (see $[\mathbf{1 6},(7.5)]$ ).

The Zassenhaus conjecture (ZC1) holds for groups $G$ which have a normal $p$-subgroup with abelian quotient (see [7, Theorem 1.2]). Thus, in the situation of Corollary 6.2 (ii), the elements $u$ and $g_{0}$ have the same order, by the remark just made. We are not aware of any obvious connection with the Whitcomb argument, which supplies a unique element $g \in G$ with $u \equiv g \bmod \mathrm{I}(\mathbb{Z} G) \mathrm{I}(\mathbb{Z} A)$ and which also has the same order as $u$ (cf. [16, (30.5), $(37.14)])$.

The following lemma is not used for the proof of Theorem 1.1, but we included it here since it is tempting to believe that it also holds for abelian $A$ when it might be combined with the approach taken in the next section to yield further results.

Lemma 6.4. Suppose that $G=X A$ with $A \unlhd G, X \leqslant G$ and with $A$ cyclic and $X$ abelian, and that (ZC1) holds for proper quotients of $G$. Let $u$ be a torsion unit in $\mathrm{V}(\mathbb{Z} G)$ with $u^{*} \neq 1$ in $\mathbb{Z} G^{*}$ (again, $G^{*}=G / \mathrm{C}_{G}(A)$ ), and let $g_{0} \in G$ be the (up to conjugacy) unique element of $G$ with $u^{*}=g_{0}^{*}$ and $\varepsilon_{g_{0}}(u)=1$ (see Corollary 6.2). Then, if $p$ is a prime such that $u^{p}$ is conjugate in $(\mathbb{Q} G)^{\times}$to an element of $G$, this element is conjugate to $g_{0}^{p}$.

Proof. Under the assumption that $u^{p}$ is rationally conjugate to an element of $G$ we must show that $\varepsilon_{g_{0}^{p}}\left(u^{p}\right) \neq 0$. Write $u=\sum_{g \in G} \lambda_{g} g\left(\right.$ all $\left.\lambda_{g} \in \mathbb{Z}\right)$. Then $u^{p} \in \sum_{g \in G} \lambda_{g} g^{p}+$ $[\mathbb{Z} G, \mathbb{Z} G]+p \mathbb{Z} G($ see $[\mathbf{1 6},(7.1)])$ and, consequently,

$$
\varepsilon_{g_{0}^{p}}\left(u^{p}\right) \equiv \sum_{g^{G}: g^{p} \sim g_{0}^{p}} \varepsilon_{g}(u) \bmod p .
$$

By Corollary 6.2 (ii), $\varepsilon_{x}(u)=0$ for all $x \in G \backslash \mathrm{C}_{G}(A)$ with $x \notin g_{0}^{G}$, so

$$
\varepsilon_{g_{0}^{p}}\left(u^{p}\right) \equiv \underbrace{\varepsilon_{g_{0}}(u)}_{=1}+\sum_{\substack{g^{G}: g^{p} \sim g_{0}^{p}, g \in \mathrm{C}_{G}(A)}} \varepsilon_{g}(u) \bmod p .
$$

We have to investigate the case when the sum that appears in equation (6.1) is nonempty, so assume that there exists an element $g_{1}$ in the normal subgroup $\mathrm{C}_{G}(A)$ of $G$ with $g_{1}^{p}=g_{0}^{p}$. Note that $\mathrm{C}_{G}(A)$ is abelian.

Suppose that $p$ divides the order of $\mathrm{C}_{G}(A)$. Set $E=\left\langle x \in \mathrm{C}_{G}(A) \mid x^{p}=1\right\rangle \neq 1$. Then $E$ is an elementary abelian normal subgroup of $G$. If $g_{2}$ is another element of $\mathrm{C}_{G}(A)$ with 
$g_{2}^{p}=g_{0}^{p}$, then $\left(g_{2} g_{1}^{-1}\right)^{p}=g_{2}^{p} g_{1}^{-p}=1$ and so $g_{2} \in E g_{1}$. Thus, the sum in (6.1) extends over the conjugacy classes of elements of $E g_{1}$. Set $\bar{G}=G / E$. By Corollary 6.2 (ii), $\bar{u} \sim \bar{g}_{0}$ in $(\mathbb{Q} \bar{G})^{\times}$, so

$$
\sum_{g^{G}: g \in E g_{1}} \varepsilon_{g}(u)=\varepsilon_{\bar{g}_{1}}(\bar{u})=0,
$$

since $\bar{g}_{1}$ and $\bar{g}_{0}$ are not conjugate in $\bar{G}$. Now it follows from (6.1) that $\varepsilon_{g_{0}^{p}}\left(u^{p}\right) \equiv 1 \bmod p$, so $\varepsilon_{g_{0}^{p}}\left(u^{p}\right) \neq 1$ as desired.

It remains to consider the case when $\mathrm{C}_{G}(A)$ is a $p^{\prime}$-group. Then $g_{1}$ is the unique element of $\mathrm{C}_{G}(A)$ with $g_{1}^{p}=g_{0}^{p}$, so $\varepsilon_{g_{0}^{p}}\left(u^{p}\right) \equiv 1+\varepsilon_{g_{1}}(u) \bmod p$ by $(6.1)$. We shall show that $g_{1} \in \mathrm{Z}(G)$, whence $\varepsilon_{g_{1}}(u)=0$ (see [16, (1.4)]) and again $\varepsilon_{g_{0}^{p}}\left(u^{p}\right) \neq 1$. We have $\left[g_{0}, g_{1}\right]=1$ since $g_{0}$ commutes with $g_{1}^{p}\left(g_{1}^{p}=g_{0}^{p}\right)$ and $\left\langle g_{1}\right\rangle=\left\langle g_{1}^{p}\right\rangle$. Thus, $g_{1}^{-1} g_{0}$ is of order $p$ and acts fixed-point freely on the cyclic $p^{\prime}$-group $A$ (this is the only place where we use the fact that $A$ is cyclic). Write $g_{1}=a x$ with $a \in A$ and $x \in \mathrm{C}_{X}(A) \subseteq \mathrm{Z}(G)$, and $g_{1}^{-1} g_{0}=b y$ with $b \in A$ and $y \in X$. Then $y$ acts fixed-point freely on $A$, so $a=1$ from $a x=g_{1}=g_{1}^{g_{1}^{-1} g_{0}}=g_{1}^{b y}=g_{1}^{y}=a^{y} x$ and $g_{1}=x \in \mathrm{Z}(G)$ as claimed.

Let us continue to suppose that $G=X A$ for $A \unlhd G, X \leqslant G$ with $A$ cyclic, $X$ abelian, and let $u$ be a torsion unit in $\mathrm{V}(\mathbb{Z} G)$ which does not map to the identity in $\mathbb{Z} G / \mathrm{C}_{G}(A)$. Also suppose that (ZC1) holds for proper quotients of $G$ and that proper subgroups of $\langle u\rangle$ are rationally conjugate to subgroups of $G$. Let $g_{0} \in G$ be defined as in Corollary 6.2. So far we have shown that then $u$ is rationally conjugate to an element of $G$ if and only if $\chi(u)=\chi\left(g_{0}\right)$ for every faithful irreducible character $\chi$ of $G$.

One might hope to prove the missing statement by some kind of 'inspection' since the irreducible $\mathbb{C}$-representations are monomial. In fact, every simple $\mathbb{C} G$-module on which $A$ acts faithfully is induced from a one-dimensional module for the abelian subgroup $\mathrm{C}_{G}(A)$ (see $[\mathbf{3},(11.1)]$ ). Thus, it remains to show that $\chi(u)=0$ for the corresponding characters $\chi$. Actually, this is equivalent to requiring that the partial augmentations of $u$ with respect to elements of $\mathrm{C}_{G}(A)$ vanish, as we will see next.

\section{Induced characters and partial augmentations}

The main result of this section is stated in Theorem 7.3. We begin with some general observations.

Let $A$ be a normal subgroup of $G$, of index $m$, say. One obtains an embedding of $R G$ into $(R A)_{m}$, the ring of $m \times m$ matrices over $R A$, by considering $R G$ as an $(R A, R G)$ bimodule, with $R G=R A \otimes_{R A} R G$. (Here $R$ is any integral domain of characteristic zero.)

To write down such an embedding explicitly, let $x_{1}, \ldots, x_{m}$ be representatives of the cosets of $A$ in $G$. Then $R G=\oplus_{j} R A x_{j}$ as $R A$-module. For $u=\sum_{g \in G} u_{g} g$ (all $u_{g}$ in $R$ ), write accordingly $x_{i} u=\sum f_{i j}(u) x_{j}$. Then $u \mapsto U=\left[f_{i j}(u)\right] \in(R A)_{m}$ defines an embedding of $R G$ into $(R A)_{m}$. (The choice of another ' $R A$-basis' would lead to a conjugate representation.) 
The relevance of this embedding with respect to the Zassenhaus conjecture (ZC1) was demonstrated in $[\mathbf{2}, \mathbf{1 3}, \mathbf{2 0}]$ and $[\mathbf{1 6}, \S 41]$. We shall be concerned only with characters, for which we recall some known facts.

The embedding exists whether or not $A$ is normal; one advantage of $A$ being normal is that traces can easily be calculated: one has $f_{i i}(u)=\left(\sum_{a \in A} u_{a} a\right)^{x_{i}^{-1}}$, so the trace of $U$ modulo $[K A, K A]$ can be expressed using the partial augmentations with respect to elements of $A$ as follows (see $[\mathbf{1 3},(2.8)])$ :

$$
\begin{aligned}
\operatorname{tr}(U) & =\sum_{i} \sum_{a \in A} u_{x_{i}^{-1} a x_{i}} a \\
& =\sum_{a^{G}: a \in A} \sum_{i} \sum_{b \in a^{G}} u_{x_{i}^{-1} b x_{i}} b \\
& \equiv \sum_{a^{G}} \sum_{i} \varepsilon_{a}(u) a \\
& \equiv \sum_{a^{G}}|G: A| \varepsilon_{a}(u) \frac{1}{\left|G: \mathrm{C}_{G}(a)\right|} \hat{\mathrm{C}}(a) \\
& =\sum_{a^{G}} \frac{\left|\mathrm{C}_{G}(a)\right|}{|A|} \varepsilon_{a}(u) \hat{\mathrm{C}}(a)
\end{aligned}
$$

where we have written $\hat{\mathcal{C}}(a)$ for the class sum of $a$, i.e. for the sum of the elements of $a^{G}$. Note that the averaged coefficients in the last sum need not lie in $R$. If one does not wish to have a congruence to an element of $K A$, one may choose, for each class $a^{G}$, elements $a_{k}$ in $A$ such that $a^{G}$ is the disjoint union of the $A$-conjugacy classes of the $a_{k}$. Then $\hat{\mathrm{C}}(a) \equiv \sum_{k}\left|A: \mathrm{C}_{A}\left(a_{k}\right)\right| a_{k}$ and

$$
\frac{\left|\mathrm{C}_{G}(a)\right|}{|A|} \varepsilon_{a}(u) \hat{\mathrm{C}}(a) \equiv \sum_{k}\left|\mathrm{C}_{G}\left(a_{k}\right): \mathrm{C}_{A}\left(a_{k}\right)\right| \varepsilon_{a_{k}}(u) a_{k}
$$

resulting in the following formula $[\mathbf{1 6},(41.10)]$ :

$$
\operatorname{tr}(U) \equiv \sum_{a^{A}: a \in A}\left|\mathrm{C}_{G}(a): \mathrm{C}_{A}(a)\right| \varepsilon_{a}(u) a \bmod [R A, R A]
$$

The given embedding $R G \rightarrow(R A)_{m}$ may be 'composed' with homomorphisms of $R A$ into some ring $\Lambda$, and also characters $R A \rightarrow K$, to obtain further homomorphisms $R G \rightarrow(\Lambda)_{m}$, and characters $K G \rightarrow K$.

Let $R=\mathbb{C}$, and let $\psi_{1}=1, \psi_{2}, \ldots, \psi_{n}$ be the irreducible complex characters of $A$. The characters $\chi_{1}, \ldots, \chi_{n}$ of $G$ one obtains in this way are just the induced characters, $\chi_{i}=\psi_{i} \uparrow^{G}$. Let $a_{1}=1, a_{2}, \ldots, a_{n}$ be representatives of the conjugacy classes of $A$, and let $\boldsymbol{Z}$ be the character table of $A$, with $(i, j)$ entry $\psi_{i}\left(a_{j}\right)$. Then (7.2) yields

$$
\left(\begin{array}{c}
\chi_{1}(u) \\
\vdots \\
\chi_{n}(u)
\end{array}\right)=Z\left(\begin{array}{c}
\left|\mathrm{C}_{G}\left(a_{1}\right): \mathrm{C}_{A}\left(a_{1}\right)\right| \varepsilon_{a_{1}}(u) \\
\vdots \\
\left|\mathrm{C}_{G}\left(a_{n}\right): \mathrm{C}_{A}\left(a_{n}\right)\right| \varepsilon_{a_{n}}(u)
\end{array}\right)
$$


It may happen that some information about the values $\chi_{i}(u)$ is available from which one would like to obtain information about the partial augmentations of $u$. For this purpose one obviously should multiply (7.3) by the inverse of $\boldsymbol{Z}$. Let $\overline{\boldsymbol{Z}}$ be the complex conjugate of $\boldsymbol{Z}$, and $\boldsymbol{Z}^{*}=\overline{\boldsymbol{Z}}^{\mathrm{T}}$ be the Hermitian transpose. Then

$$
\boldsymbol{Z}^{*} \boldsymbol{Z}=|A| \cdot \operatorname{diag}\left(\left|A: \mathrm{C}_{A}\left(a_{1}\right)\right|, \ldots,\left|A: \mathrm{C}_{A}\left(a_{n}\right)\right|\right)^{-1}
$$

(see $[\mathbf{3},(9.26)])$, and it follows that

$$
\left(\chi_{1}(u), \ldots, \chi_{n}(u)\right) \overline{\boldsymbol{Z}}=\left(\left|\mathrm{C}_{G}\left(a_{1}\right)\right| \varepsilon_{a_{1}}(u), \ldots,\left|\mathrm{C}_{G}\left(a_{n}\right)\right| \varepsilon_{a_{n}}(u)\right) .
$$

What kind of information may we have about the $\chi_{i}(u)$ ? Of course, we are thinking of $u$ as a torsion unit, when it should be appreciated that the $\chi_{i}(u)$ are sums of roots of unity. Equation (7.3) itself may provide information.

Remark 7.1. Let $u$ be a torsion unit in $\mathrm{V}(\mathbb{Z} G)$ which does not map to the identity in $\mathbb{Z} G / A$, and suppose that (ZC1) holds for proper quotients of $G$. Then $\chi_{i}(u)=0$ if the kernel of the character $\psi_{i}$ contains a non-trivial normal subgroup of $G$.

Proof. Let $B$ be a non-trivial normal subgroup of $G$ contained in $A$, and set $\bar{G}=G / B$. If $B$ lies in the kernel of some irreducible character $\psi_{i}$ of $A$, then $\psi_{i}$ is inflated from an irreducible character $\varphi$ of $\bar{A}$, and $\chi_{i}$ is inflated from $\varphi \uparrow \bar{G}$. Thus, we have to show that $\varphi \uparrow^{\bar{G}}(\bar{u})=0$ for the image $\bar{u}$ of $u$ in $\mathbb{Z} \bar{G}$. Since (ZC1) holds for $\bar{G}$ and $\bar{u}$ does not map to the identity in $\mathbb{Z} \bar{G} / \bar{A}$, we have $\varepsilon_{\bar{a}}(\bar{u})=0$ for all $a \in A$. Therefore, the claim follows from (7.3) applied to the group $\bar{G}$, its normal subgroup $\bar{A}$ and the element $\bar{u}$.

We merely note down that (7.4) is compatible with any group action on $A$, in the following sense.

Remark 7.2. Suppose that $\mathcal{G}$ is a group acting on $A$. Then $\mathcal{G}$ also acts on the irreducible characters $\operatorname{Irr}_{\mathbb{C}}(A)$ if one defines $\psi^{\sigma}$, for $\psi \in \operatorname{Irr}_{\mathbb{C}}(A)$ and $\sigma \in \mathcal{G}$, by setting $\psi^{\sigma}(a)=\psi\left(a^{\sigma^{-1}}\right)$. This action is compatible with the action on the conjugacy classes of $A$ in the following sense (cf. Brauer's 'permutation lemma' [3, (11.9)]). For $\sigma \in \mathcal{G}$, define $m \times m$ permutation matrices $\boldsymbol{P}_{\text {col }}(\sigma)$ and $\boldsymbol{P}_{\text {row }}(\sigma)$ by requiring that $\boldsymbol{P}_{\text {col }}(\sigma)_{i, j}=1$ if $\left(a_{i}^{\sigma}\right)^{A}=a_{j}^{A}$ and $\boldsymbol{P}_{\text {row }}(\sigma)_{i, j}=1$ if $\psi_{i}^{\sigma}=\psi_{j}$. Then the $i$ th column of $\boldsymbol{Z} \boldsymbol{P}_{\text {col }}(\sigma)$ is the column of $\boldsymbol{Z}$ corresponding to the class of $a_{i}^{\sigma}$, and the $i$ th row of $\boldsymbol{P}_{\text {row }}(\sigma) \boldsymbol{Z}$ is the row of $\boldsymbol{Z}$ corresponding to the class of $\psi_{i}^{\sigma}$. It follows that $\boldsymbol{Z} \boldsymbol{P}_{\text {col }}(\sigma)=\boldsymbol{P}_{\text {row }}\left(\sigma^{-1}\right) \boldsymbol{Z}$. (And the permutation representations $\sigma \mapsto \boldsymbol{P}_{\text {col }}(\sigma)$ and $\sigma \mapsto \boldsymbol{P}_{\text {row }}\left(\sigma^{-1}\right)$ of $\mathcal{G}$ are conjugate.)

The group $\mathcal{G}$ acts on the induced characters, $\chi_{i}^{\sigma}=\psi_{i}^{\sigma} \uparrow G$. Replacing $\boldsymbol{Z}$ by $\boldsymbol{P}_{\text {row }}(\sigma) \boldsymbol{Z} \boldsymbol{P}_{\text {col }}(\sigma)$ in (7.3) gives us

$$
\left(\chi_{1}^{\sigma^{-1}}(u), \ldots, \chi_{n}^{\sigma^{-1}}(u)\right) \overline{\boldsymbol{Z}}=\left(\left|\mathrm{C}_{G}\left(a_{1}^{\sigma}\right)\right| \varepsilon_{a_{1}^{\sigma}}(u), \ldots,\left|\mathrm{C}_{G}\left(a_{n}^{\sigma}\right)\right| \varepsilon_{a_{n}^{\sigma}}(u)\right) .
$$

Perhaps this can be helpful to obtain relations between the $\varepsilon_{a_{i}^{\sigma}}(u), \sigma \in \mathcal{G}$, when $u$ is a torsion unit in $\mathbb{Z} G$.

In the special case when $A$ is cyclic, we can prove, with the help of (7.4), the following theorem. 
Theorem 7.3. Let $A$ be a cyclic normal subgroup of $G$. Suppose that

(i) (ZC1) holds for proper quotients of $G$;

(ii) every torsion unit in $\mathrm{V}(\mathbb{Z} G)$ which maps to the identity in $\mathbb{Z} G / A$ is rationally conjugate to an element of $G$.

Then for each torsion unit $u$ in $\mathrm{V}(\mathbb{Z} G)$ which does not map to the identity in $\mathbb{Z} G / A$, all partial augmentations $\varepsilon_{a}(u), a \in A$, are zero.

Proof. We keep previously introduced notation. Let $\zeta$ be a primitive $n$th root of unity $($ now $n=|A|)$, and set $\mathcal{G}=\operatorname{Gal}(\mathbb{Q}(\zeta) / \mathbb{Q})$. For $\sigma \in \mathcal{G}$, we have $\zeta^{\sigma}=\zeta^{i}$ for some integer $i=i(\sigma)$, uniquely determined modulo $n$. The map $\sigma \mapsto i(\sigma)$ gives an isomorphism $\mathcal{G} \rightarrow(\mathbb{Z} / n \mathbb{Z})^{\times}$(see $[\mathbf{1 1}, \S$ VI, Theorem 3.1]). Thus, $\mathcal{G}$ acts on $A$ as the full automorphism group, $a^{\sigma}=a^{i(\sigma)}$ for $a \in A$. The action of $\mathcal{G}$ on the irreducible characters of $A$-according to Remark 7.2-is described by $\psi^{\sigma^{-1}}(a)=\psi\left(a^{\sigma}\right)=\psi\left(a^{i(\sigma)}\right)=\psi(a)^{i(\sigma)}=\psi(a)^{\sigma}$, for $\psi \in$ $\operatorname{Irr}_{\mathbb{C}}(A), a \in A$. Note that also $\psi^{\sigma^{-1}}(v)=\psi(v)^{\sigma}$ and $(\psi \uparrow G)^{\sigma^{-1}}(u)=\left(\psi \uparrow \uparrow^{G}\right)(u)^{\sigma}$ for $\psi \in$ $\operatorname{Irr}_{\mathbb{C}}(A), v \in \mathbb{Q} A$ and $u \in \mathbb{Q} G$.

Let $u$ be a torsion unit in $\mathrm{V}(\mathbb{Z} G)$ which does not map to the identity in $\mathbb{Z} G / A$. The essential observation will be that the entries of $\left(\chi_{1}(u), \ldots, \chi_{n}(u)\right) \bar{Z}$ have a simple description in terms of the Galois action. Fix a faithful irreducible character $\psi$ of $A$ and set $\chi=\psi \uparrow^{G}$. The Galois conjugates of $\psi$ are all distinct and give the faithful irreducibles. It follows from Remark 7.1 that, for $a \in A$,

$$
\sum_{i=1}^{n} \chi_{i}(u) \overline{\psi_{i}(a)}=\sum_{\sigma \in \mathcal{G}} \chi^{\sigma^{-1}}(u) \overline{\psi^{\sigma^{-1}}(a)}=\sum_{\sigma \in \mathcal{G}} \chi(u)^{\sigma} \overline{\psi(a)^{\sigma}} .
$$

Thus, (7.4) means that

$$
\operatorname{Tr}_{\mathbb{Q}(\zeta) / \mathbb{Q}}(\chi(u) \overline{\psi(a)})=\left|\mathrm{C}_{G}(a)\right| \varepsilon_{a}(u) \text { for all } a \in A .
$$

We shall use induction on the number of prime factors on the order of $u$. Thus, to start the induction, $u$ will be of prime order, but we will treat this case simultaneously with the general induction step. Suppose by way of contradiction that $\varepsilon_{a}(u) \neq 0$ for some $a \in A$. We shall show that $\varepsilon_{a}(u) \geqslant 0$. Since the image of $u$ in $\mathbb{Z} G / A$ has vanishing 1-coefficient (see $[\mathbf{1 6},(1.5)]$ ), this will prove the theorem.

Set $\xi=\psi(a)$. Let $e$ denote the order of $u$, and let $\theta$ be a primitive $e$ th root of unity. Since the order of $a$ divides $e$ (see Remark 2.4), we have $\xi \in \mathbb{Q}(\theta)$.

Let $\chi$ be afforded by a representation $D$, and write $\mu(\xi, u, \chi)$ for the multiplicity of the eth root of unity $\xi$ as an eigenvalue of $D(u)$. Then (see Remark 2.5)

$$
\mu(\xi, u, \chi)=\frac{1}{e} \sum_{d \mid e} \operatorname{Tr}_{\mathbb{Q}\left(\theta^{d}\right) / \mathbb{Q}}\left(\chi\left(u^{d}\right) \xi^{-d}\right) .
$$

We shall use (7.5) to simplify this sum. Let $f$ denote the order of the image of $u$ in $\mathrm{V}(\mathbb{Z} G / A)$. Let $d \mid e$ with $f \nmid d$, so that $u^{d}$ does not map to the identity in $\mathbb{Z} G / A$. If 
$d \neq 1$, then $u$ is not of prime order, and we can inductively assume that $\varepsilon_{a}\left(u^{d}\right)=0$ for all $a \in A$. Taking traces is transitive (see $[\mathbf{1 1}, \S \mathrm{VI}$, Theorem 5.1]), so

$$
\left[\mathbb{Q}\left(\zeta, \theta^{d}\right): \mathbb{Q}\left(\theta^{d}\right)\right] \operatorname{Tr}_{\mathbb{Q}\left(\theta^{d}\right) / \mathbb{Q}}\left(\chi\left(u^{d}\right) \xi^{-d}\right)=\left[\mathbb{Q}\left(\zeta, \theta^{d}\right): \mathbb{Q}(\zeta)\right] \operatorname{Tr}_{\mathbb{Q}(\zeta) / \mathbb{Q}}\left(\chi\left(u^{d}\right) \xi^{-d}\right)
$$

or

$$
\operatorname{Tr}_{\mathbb{Q}\left(\theta^{d}\right) / \mathbb{Q}}\left(\chi\left(u^{d}\right) \xi^{-d}\right)=\frac{\left[\mathbb{Q}\left(\theta^{d}\right): \mathbb{Q}\right]}{[\mathbb{Q}(\zeta): \mathbb{Q}]} \operatorname{Tr}_{\mathbb{Q}(\zeta) / \mathbb{Q}}\left(\chi\left(u^{d}\right) \xi^{-d}\right)
$$

Note that (7.5) also holds with $u$ replaced by $u^{d}$ (and $a$ replaced by $a^{d}$ ), so the inductive step shows that $\operatorname{Tr}_{\mathbb{Q}\left(\theta^{d}\right) / \mathbb{Q}}\left(\chi\left(u^{d}\right) \xi^{-d}\right)=0$ if $d \neq 1$. When $d=1$, (7.5), now slightly modified, reads

$$
\operatorname{Tr}_{\mathbb{Q}(\theta) / \mathbb{Q}}\left(\chi(u) \xi^{-1}\right)=\frac{\varphi(e)}{\varphi(|A|)}\left|\mathrm{C}_{G}(a)\right| \varepsilon_{a}(u),
$$

where $\varphi$ denotes Euler's phi function. Furthermore,

$$
\begin{aligned}
\frac{1}{e} \sum_{d|e: f| d} \operatorname{Tr}_{\mathbb{Q}\left(\theta^{d}\right) / \mathbb{Q}}\left(\chi\left(u^{d}\right) \xi^{-d}\right) & =\frac{1}{e} \sum_{d \mid e / f} \operatorname{Tr}_{\mathbb{Q}\left(\left(\theta^{f}\right)^{d}\right) / \mathbb{Q}}\left(\chi\left(\left(u^{f}\right)^{d}\right) \xi^{-f d}\right) \\
& =\frac{1}{f} \mu\left(\xi^{f}, u^{f}, \chi\right) .
\end{aligned}
$$

Taken altogether,

$$
\mu(\xi, u, \chi)=\frac{1}{f} \mu\left(\xi^{f}, u^{f}, \chi\right)+\frac{1}{e} \frac{\varphi(e)}{\varphi(|A|)}\left|\mathrm{C}_{G}(a)\right| \varepsilon_{a}(u) .
$$

We remark for later use that, when $f$ is even, (7.6) holds with $\xi$ replaced by $-\xi$ and it follows in the same way that

$$
\mu(-\xi, u, \chi)=\frac{1}{f} \mu\left(\xi^{f}, u^{f}, \chi\right)-\frac{1}{e} \frac{\varphi(e)}{\varphi(|A|)}\left|\mathrm{C}_{G}(a)\right| \varepsilon_{a}(u) .
$$

Note that $\mu(\xi, u, \chi)$ and $\mu\left(\xi^{f}, u^{f}, \chi\right)$ are non-negative (integers). We can assume that $\mu\left(\xi^{f}, u^{f}, \chi\right) \neq 0$ since otherwise obviously $\varepsilon_{a}(u) \geqslant 0$. By assumption (ii), $u^{f}$ is rationally conjugate to an element $b$ of $A$. The eigenvalues of the matrix $D(b)$ (remember that $D$ is a representation affording the induced character $\chi)$ are the roots of unity $\psi\left(b^{g_{i}}\right)$ where $g_{1}, \ldots, g_{r}$ are representatives of the cosets of $\mathrm{C}_{G}(b)$ in $G$, each one occurring with multiplicity $\chi(1) /\left|G: \mathrm{C}_{G}(b)\right|$. Since $\xi^{f}$ is an eigenvalue, $\psi\left(a^{f}\right)=\xi^{f}=\psi\left(b^{g_{i}}\right)$ for some index $i$. It follows that $u^{f}$ is rationally conjugate to $a^{f}$, and

$$
\frac{1}{f} \mu\left(\xi^{f}, u^{f}, \chi\right)=\frac{1}{f} \frac{\left|\mathrm{C}_{G}\left(a^{f}\right)\right|}{|A|} .
$$

Again, we note for later use that if $\sigma_{i} \in \mathcal{G}$ are such that $\psi\left(a^{g_{i}}\right)=\xi^{\sigma_{i}}$ (remember that $\xi=\psi(a))$ and $f$ is even, then (7.8) holds with $\xi$ replaced by $\xi^{\sigma_{i}}$, and so

$$
\mu\left(-\xi^{\sigma_{1}}, u, \chi\right)=\cdots=\mu\left(-\xi^{\sigma_{r}}, u, \chi\right) .
$$


We will show that

$$
\frac{1}{f} \frac{\left|\mathrm{C}_{G}\left(a^{f}\right)\right|}{|A|} \leqslant \frac{1}{e} \frac{\varphi(e)}{\varphi(|A|)}\left|\mathrm{C}_{G}(a)\right|
$$

where equality can hold only when $f=2$ and $|A|$ is odd. Thus, in the end, only this case will be left to consider since strict inequality implies by (7.7) and (7.9) that the rational integer $\varepsilon_{a}(u)$ is non-negative.

Write $f=f_{1} f_{2}$ such that the prime divisors of $f_{1}$ divide $|A|$ and $\left(f_{2},|A|\right)=1$. Since $e / f$ is the order of $a^{f}$, we have $e=f_{2} e^{\prime}$ with all prime divisors of $e^{\prime}$ dividing $|A|$. Remembering that $\varphi(k) / k=\prod_{i=1}^{s}\left(1-1 / p_{i}\right)$ for a natural number $k$ whose distinct prime divisors are $p_{1}, \ldots, p_{s}$, we obtain

$$
f_{2} \frac{1}{e} \frac{\varphi(e)}{\varphi(|A|)}=\frac{1}{e^{\prime}} \frac{\varphi\left(f_{2}\right) \varphi\left(e^{\prime}\right)}{\varphi(|A|)}=\varphi\left(f_{2}\right) \underbrace{\frac{\varphi\left(e^{\prime}\right) / e^{\prime}}{\varphi(|A|) /|A|}}_{\geqslant 1} \frac{1}{|A|} \geqslant \frac{1}{|A|} .
$$

Note that equality can only hold if $f_{2} \leqslant 2$.

Set $Q=\mathrm{C}_{G}\left(a^{f}\right) / \mathrm{C}_{G}(a)$. We proceed to show that $|Q| \leqslant f_{1}$ (a general fact) and that $|Q|<f_{1}$ if $f_{1} \neq 1$. Together with the last inequality, this establishes (7.11):

$$
\frac{1}{f_{1}} \frac{\left|\mathrm{C}_{G}\left(a^{f}\right)\right|}{|A|} \leqslant \frac{\left|\mathrm{C}_{G}(a)\right|}{|A|} \leqslant f_{2} \frac{1}{e} \frac{\varphi(e)}{\varphi(|A|)}\left|\mathrm{C}_{G}(a)\right|
$$

with equality both times possible only if $f_{1}=1$ and $f_{2}=2$ (remember that $f \neq 1$ ).

Note that $\left\langle a^{f}\right\rangle=\left\langle a^{f_{1}}\right\rangle$ and so $\mathrm{C}_{G}\left(a^{f}\right)=\mathrm{C}_{G}\left(a^{f_{1}}\right)$. The quotient $Q$ is the kernel of the natural surjection $G / \mathrm{C}_{G}(a) \rightarrow G / \mathrm{C}_{G}\left(a^{f_{1}}\right)$. When $G / \mathrm{C}_{G}(a)$ is viewed as a subgroup of $\operatorname{Aut}(\langle a\rangle), Q$ consists of automorphisms fixing $a^{f_{1}}$. Thus, $|Q| \leqslant f_{1}$ (both this fact and next are described in detail separately in Claim 7.4, below). Suppose that $|Q|=f_{1} \neq 1$. Then prime divisors of $f_{1}$ are prime divisors of $|\langle a\rangle|$, and if we set $B=\langle b \in\langle a\rangle| b^{p}=1$ for some $\left.p \in \pi\left(f_{1}\right)\right\rangle$, then $1 \neq B \unlhd G$ and $B a \subseteq a^{G}$. Thus, if we set $\bar{G}=G / B$ and $\bar{u}$ denotes, as usual, the image of $u$ in $\mathbb{Z} \bar{G}$, then $\varepsilon_{\bar{a}}(\bar{u})=\varepsilon_{a}(u) \neq 0$. But this contradicts the assumption that (ZC1) holds for $\bar{G}$ and $\bar{u}$ does not map to the identity in $\mathbb{Z} \bar{G} / \bar{A}$. Therefore, $|Q|<f_{1}$ if $f_{1} \neq 1$.

Finally, we consider the case when $f=2$ and $|A|$ is odd. It follows from (7.7), (7.9) and (7.11) that either $\varepsilon_{a}(u) \geqslant 0$ or $\varepsilon_{a}(u)=-1$, and in the latter case the supplements (7.8) and (7.10) provide us with $\mu\left(-\xi^{\sigma_{i}}, u, \chi\right)=\mu\left(\xi^{2}, u^{2}, \chi\right)=\left|\mathrm{C}_{G}\left(a^{2}\right)\right| /|A|$ for $i=1,2, \ldots, r=\left|G: \mathrm{C}_{G}\left(a^{2}\right)\right|$. Now, suppose that $\varepsilon_{a}(u)=-1$. Since the $\left(\xi^{2}\right)^{\sigma_{i}}$ are the distinct eigenvalues of $D\left(u^{2}\right)$ and $\chi(1)=|G: A|$, it follows that the $-\xi^{\sigma_{i}}$ are the distinct eigenvalues of $D(u)$. Thus, $D\left(u^{e / 2}\right)$ is the negative of the identity matrix. Since $u^{e / 2}$ has order $2, A$ is of odd order and ( $\mathrm{ZC} 1)$ holds for $G / A$, we know that $u^{e / 2}$ is rationally conjugate to an element $z$ of $G$ (see [5, Theorem 2.2]). It follows that $D\left(u^{e / 2}\right)=D(z)$, and $z \in \mathrm{Z}(G)$, since $D$ is a faithful representation of $G$. Thus, $z$ is the 2-part of $u$, and $z^{-1} u$ is a torsion unit in $\mathrm{V}(\mathbb{Z} G)$ which maps to the identity in $\mathbb{Z} G / A$. By assumption (ii), the partial augmentations of $z^{-1} u$ are non-negative, in contradiction to $\varepsilon_{z^{-1} a}\left(z^{-1} u\right)=\varepsilon_{a}(u)=-1$. The proof of the theorem is complete. 
We append an elementary fact used in the above proof. We write $\pi(n)$ for the set of prime divisors of a natural number $n$.

Claim 7.4. Let $X$ be a cyclic group, let $k \in \mathbb{N}$ and let $Q$ be the subgroup of $\operatorname{Aut}(X)$, fixing $X^{k}$ element-wise. Then $|Q| \leqslant k$. Furthermore, if $k \neq 1$ and $|Q|=k$, then $\pi(k) \subseteq$ $\pi(|X|)$ and, for $B=\langle x \in X| x^{p}=1$ for some $\left.p \in \pi(k)\right\rangle$ and a generator $a$ of $X$, the coset $B a$ is contained in a single orbit under the action of $Q$ on $X$.

Proof. Let $X_{i}(i \in I)$ be the Sylow subgroups of $X$. Then $\operatorname{Aut}(X)=\prod_{i \in I} \operatorname{Aut}\left(X_{i}\right)$ and $Q=\prod_{i \in I} Q_{i}$, where $Q_{i}=\left\langle\alpha \in \operatorname{Aut}\left(X_{i}\right)\right| \alpha$ fixes $X_{i}^{k}$ element-wise $\rangle$. This will reduce the proof of the claim to the case where $X$ is a $p$-group.

So, assume for the moment that $|X|=p^{s}$ for some prime $p$ and $s \geqslant 1$. Let $p^{t}$ be the $p$-part of $k$. Then one of the following holds:

(i) $p^{t}=1$, when $|Q|=1$;

(ii) $t \geqslant s$, i.e. $X^{k}=1$, when $|Q|=|\operatorname{Aut}(X)|=(p-1) p^{s-1}<p^{s} \leqslant p^{t}$;

(iii) $1 \leqslant t<s$, when $|Q|=p^{t}$ (since $\left|X^{k}\right|=p^{s-t} \neq 1$ and $Q$ is isomorphic to the kernel of the surjection $\left.\left(\mathbb{Z} / p^{s} \mathbb{Z}\right)^{\times} \rightarrow\left(\mathbb{Z} / p^{s-t} \mathbb{Z}\right)^{\times}\right)$.

Note that, in any case, $|Q|$ is less than or equal to the $p$-part of $k$.

Returning to the general case, it follows that $|Q| \leqslant k$. Suppose that $k \neq 1$ and $|Q|=k$. Then $\pi(k) \subseteq \pi(|X|)$. Let $p \in \pi(k)$ and let $X_{i(p)}$ be the Sylow $p$-subgroup of $X$, with generator $x_{p}$. Then we are in situation (iii), i.e. the $p$-part of $k$ is less than the order of $x_{p}$. If $b_{p}$ denotes an element of order $p$ in $X_{i(p)}$, then $\alpha_{p}: x_{p} \mapsto x_{p} b_{p}$ defines an automorphism of $X_{i(p)}$ of order $p$ which can be viewed as an automorphism of $X$, and then $\alpha_{p} \in Q$. Now if $Q_{0}=\left\langle\alpha_{p} \mid p \in \pi(k)\right\rangle \leqslant Q, B=\left\langle b_{p} \mid p \in \pi(k)\right\rangle \leqslant X$ and $X=\langle a\rangle$, then $B a=\left\{a \gamma \mid \gamma \in Q_{0}\right\}$. This completes the proof.

Now we can complete the proof of Theorem 1.1.

Corollary 7.5. Suppose that $G=X A$ with $A \unlhd G, X \leqslant G$ and with $A$ cyclic and $X$ abelian. Set $G^{*}=G / \mathrm{C}_{G}(A)$ and suppose that $u^{*} \neq 1$ in $\mathbb{Z} G^{*}$ for a torsion unit $u$ in $\mathrm{V}(\mathbb{Z} G)$. Then $u$ is rationally conjugate to an element of $G$.

Proof. By induction on the order of $G$ and in view of Corollary 5.3 we can assume that (ZC1) holds for proper quotients of $G$. Also Theorem 7.3 (ii) holds by Corollary 5.3. Thus, by Theorem 7.3, all partial augmentations $\varepsilon_{a}(u), a \in A$, are zero.

Let $g \in \mathrm{C}_{G}(A)$. We can write $g=x a$ with $x \in \mathrm{C}_{X}(A)$ and $a \in A$. Note that $x \in \mathrm{Z}(G)$, so $x^{-1} u$ is a torsion unit with $\left(x^{-1} u\right)^{*} \neq 1$ and $\varepsilon_{g}(u)=\varepsilon_{a}\left(x^{-1} u\right)=0$ by the previous paragraph.

By Corollary $6.2, \varepsilon_{g}(u) \in\{0,1\}$ for all $g \in G \backslash \mathrm{C}_{G}(A)$.

Thus, all but one of the partial augmentations of $u$ vanish. Again using Corollary 5.3, it follows that the same holds for all powers of $u$, and therefore $u$ is rationally conjugate to an element of $G$ (see $[\mathbf{1 6},(41.5)]$ ). 


\section{Zassenhaus conjecture (ZC1) for some direct products}

It is hard to predict how the Zassenhaus conjecture $(\mathrm{ZC} 1)$ behaves under taking direct products of groups for which (ZC1) holds (in fact, almost nothing is known in this direction, cf. [1]). Finally, we present two positive results.

The results from $\S 3$ can be used to prove an extension of a result of Weiss (see $[\mathbf{2 1}$, Corollary], which is the $G=1$ case of the following proposition) in an alternative way. We also give an obvious extension of a result of Höfert [9], which was the source of inspiration for the approach taken in $\S 7$.

Proposition 8.1. Let $G$ be a finite group for which (ZC1) holds, and let $\Pi$ be a nilpotent group with $(|G|,|\Pi|)=1$. Then (ZC1) holds for the direct product $G \times \Pi$. Actually, a torsion unit in $\mathrm{V}(\mathbb{Z}(G \times \Pi))$ is conjugate to an element of $G \times \Pi$ already in the units of $S(G \times \Pi)$, where $S$ is the semi-localization $\mathbb{Z}_{\pi(|\Pi|)}$.

Proof. Set $P=G \times \Pi$ and let $u$ be a torsion unit in $\mathrm{V}(\mathbb{Z} P)$. If $p$ is a prime divisor of $|\Pi|$, then the $p$-part of $u$ is conjugate in $\left(\mathbb{Z}_{p} P\right)^{\times}$to an element $x_{p}$ of the Sylow $p$-subgroup of $\Pi$, by Corollary 3.3. Choose such elements $x_{p}$, one for each prime divisor $p$, and let $x$ be their product. By Lemma 2.2, $\varepsilon_{a}(u)=0$ for all $a$ in $P$ whose $|\Pi|$-part is not conjugate to $x$. Thus, we can choose $g_{1}, \ldots, g_{s} \in G$ such that the $\varepsilon_{g_{i} x}(u)$ are the distinct non-vanishing partial augmentations of $u$. It follows that the image $\bar{u}$ of $u$ under the homomorphism $\mathbb{Z} P \rightarrow \mathbb{Z} \bar{P}=\mathbb{Z} P / \Pi$ has distinct non-vanishing partial augmentations $\varepsilon_{\bar{g}_{i}}(\bar{u})$, and hence precisely one partial augmentation of $u$ does not vanish, since (ZC1) holds for $G=P / \Pi$ by assumption. Thus, so far we have shown that (ZC1) holds for $P$ and that $u$ is rationally conjugate to an element $a$ of $P$.

Let $p$ be a prime divisor of $|\Pi|$. It remains to show that $u$ is conjugate to $a$ in $\left(\mathbb{Z}_{p} P\right)^{\times}$ (see $[\mathbf{3},(31.15)])$. Let $u_{p}, u_{p^{\prime}}$ and $a_{p}, a_{p^{\prime}}$ be the $p$ - and $p^{\prime}$-parts of $u$ and $a$, respectively. There exists $v \in\left(\mathbb{Z}_{p} P\right)^{\times}$such that $u_{p^{\prime}}^{v}=a_{p^{\prime}}$ (see [7, Lemma 2.9]). We noted at the beginning of the proof that we can choose $w \in\left(\mathbb{Z}_{p} P\right)^{\times}$such that $u_{p}^{v w}=a_{p}$. Set $N=\mathrm{O}_{p}(P)$. Since $P=N \times \mathrm{O}_{p^{\prime}}(P)$, we can choose $w$ to lie in $1+\mathrm{I}\left(\mathbb{Z}_{p} N\right) G$. Then $u^{v w} \equiv a \bmod \mathrm{I}\left(\mathbb{Z}_{p} N\right) P$. Thus, if we set $\tilde{u}=u^{v w}$ and let $m$ denote the order of $a_{p^{\prime}}$, then $k=(1 / m) \sum_{i=0}^{m-1} \tilde{u}^{i} a^{-i} \in 1+\mathrm{I}\left(\mathbb{Z}_{p} N\right) P$. We have $1+\mathrm{I}\left(\mathbb{Z}_{p} N\right) P \subseteq\left(\mathbb{Z}_{p} P\right)^{\times}$ (see $[\mathbf{3},(5.26)])$. Moreover, if $n$ denotes the order of $a$, then $k=(1 / n) \sum_{i=0}^{n-1} \tilde{u}^{i} a^{-i}$ as $\tilde{u}^{m} a^{-m}=1$, so $\tilde{u} k a^{-1}=k$ and $u^{v w k}=a$.

Höfert proved the following result in $[\mathbf{9}]$ for $A$ of prime order, and it was announced in [10, Proposition 3.2] for $A$ an elementary abelian group.

Proposition 8.2. Let $A$ be a finite abelian group of exponent $m$, and let $\zeta$ be a primitive mth root of unity. Suppose that any torsion unit in $\mathrm{V}(\mathbb{Z}[\zeta] G)$ is conjugate in $(\mathbb{Q}(\zeta) G)^{\times}$to an element of $G$. Then the Zassenhaus conjecture (ZC1) holds for $G \times A$.

Proof. Let $u$ be a torsion unit in $\mathrm{V}(\mathbb{Z}(G \times A))$; we shall show that all but one of the partial augmentations of $u$ vanish. Note that $\mathbb{Z}(G \times A)=\mathbb{Z} G \otimes_{\mathbb{Z}} \mathbb{Z} A$. The unit $u$ maps under the homomorphism $\varepsilon \otimes$ id $: \mathbb{Z}(G \times A) \rightarrow \mathbb{Z} A$ to an element $a$ of $A$, by a result of G. Higman. Replacing $u$ by $u a^{-1}$, we can assume that $u$ maps to the identity. 
Let $\chi$ be an irreducible complex character of $A$. We have a commutative diagram of ring homomorphisms:

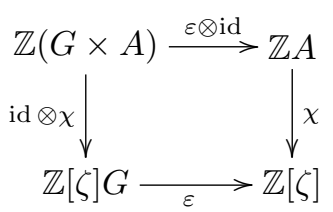

showing that $u$ maps under $\varphi=\mathrm{id} \otimes \chi$ to a unit $u \varphi$ of augmentation 1 in $\mathbb{Z}[\zeta] G$. By assumption, $\varepsilon_{g}(u \varphi)=1$ for the elements $g$ of a unique conjugacy class of $G$, and the other partial augmentations of $u \varphi$ vanish. Note that $\varepsilon_{g}(u \varphi)=\sum_{a \in A} \chi(a) \varepsilon_{g a}(u)$ for all $g \in G$.

Let $a_{1}=1, a_{2}, \ldots, a_{n}$ be the elements of $A$, and let $\chi_{1}=\mathrm{id}, \chi_{2}, \ldots, \chi_{n}$ be the irreducible complex characters of $A$. Let $\boldsymbol{Z}$ be the character table of $A$, with $(i, j)$ entry $\chi_{i}\left(a_{j}\right)$, and $\boldsymbol{Z}^{*}$ its Hermitian transpose, so that $\boldsymbol{Z}^{*} \boldsymbol{Z}=|A| \boldsymbol{I}_{n}$ where $\boldsymbol{I}_{n}$ is the $n \times n$ identity matrix. Set $\varphi_{i}=\operatorname{id} \otimes \chi_{i}: \mathbb{Z}(G \times A) \rightarrow \mathbb{Z}[\zeta] G$.

Take any $g \in G$, and set

$$
\boldsymbol{x}=\left(\varepsilon_{g a_{1}}(u), \ldots, \varepsilon_{g a_{n}}(u)\right), \quad \boldsymbol{y}=\left(\varepsilon_{g}\left(u \varphi_{1}\right), \ldots, \varepsilon_{g}\left(u \varphi_{n}\right)\right) .
$$

Then $\boldsymbol{y}$ is a vector with $0 / 1$ entries, and $\boldsymbol{y}^{\mathrm{T}}=\boldsymbol{Z} \boldsymbol{x}^{\mathrm{T}}$. From this we obtain $|A| \boldsymbol{x}^{\mathrm{T}}=\boldsymbol{Z}^{*} \boldsymbol{y}^{\mathrm{T}}$, where the entries of the vector on the left-hand side lie in $|A| \mathbb{Z}$, and those on the righthand side are sums of roots of unity taken from a column of $\boldsymbol{Z}$. It follows that either $\boldsymbol{x}=0$ or $\boldsymbol{y}=(1,1, \ldots, 1)$ and, in the latter case, $\boldsymbol{x}=(1,0, \ldots, 0)$. Thus, all partial augmentations of $u$ are either 0 or 1 and, since they add up to 1 , we are done.

\section{References}

1. V. Bovdi, C. Höfert And W. Kimmerle, On the first Zassenhaus conjecture for integral group rings, Publ. Math. Debrecen 65 (2004), 291-303.

2. G. Cliff AND A. Weiss, Finite groups of matrices over group rings, Trans. Am. Math. Soc. 352 (2000), 457-475.

3. C. W. CURTIS AND I. ReIneR, Methods of representation theory: with applications to finite groups and orders, Volume I, Pure and Applied Mathematics (Wiley, 1981).

4. Á. Del Río and S. K. SehGal, Zassenhaus conjecture (ZC1) on torsion units of integral group rings for some metabelian groups, Arch. Math. 86 (2006), 392-397.

5. M. A. Dokuchaev and S. O. JuriaAns, Finite subgroups in integral group rings, Can. J. Math. 48 (1996), 1170-1179.

6. M. HeRtweck, Local analysis of the normalizer problem, J. Pure Appl. Alg. 163 (2001), 259-276.

7. M. Hertweck, On the torsion units of some integral group rings, Algebra Colloq. $\mathbf{1 3}$ (2006), 329-348.

8. M. Hertweck, Partial augmentations and Brauer character values of torsion units in group rings, Commun. Alg., in the press.

9. C. HöFERT, Die erste Vermutung von Zassenhaus für Gruppen kleiner Ordnung, Master's thesis, University of Stuttgart (2004).

10. C. HöFERT AND W. KimmerLe, On torsion units of integral group rings of groups of small order, Lecture Notes in Pure and Applied Mathematics, Volume 248, pp. 243-252 (CRC Press/Chapman and Hall, 2006). 
11. S. LANG, Algebra, 3rd edn, Graduate Texts in Mathematics, Volume 211 (Springer, 2002).

12. I. S. Luthar And I. B. S. Passi, Zassenhaus conjecture for $A_{5}$, Proc. Indian Acad. Sci. Math. Sci. 99 (1989), 1-5.

13. Z. Marciniak, J. Ritter, S. K. Sehgal and A. Weiss, Torsion units in integral group rings of some metabelian groups, II, J. Number Theory 25 (1987), 340-352.

14. D. S. PASSMAn, The algebraic structure of group rings, Pure and Applied Mathematics (Wiley Interscience, 1977).

15. C. Polcino Milies, J. Ritter and S. K. Sehgal, On a conjecture of Zassenhaus on torsion units in integral group rings, II, Proc. Am. Math. Soc. 97 (1986), 201-206.

16. S. K. Sehgal, Units in integral group rings, Pitman Monographs and Surveys in Pure and Applied Mathematics, Volume 69 (Longman, Harlow, 1993).

17. S. K. SehGal, Zassenhaus conjecture, Encyclopaedia of mathematics, Supplement Volume III, pp. 453-454 (Kluwer, Dordrecht, 2002).

18. S. K. SEHGAL, Group rings, Handbook of algebra, Volume 3, pp. 455-541 (North-Holland, Amsterdam, 2003).

19. J. ThÉvenaz, G-algebras and modular representation theory, Oxford Mathematical Monographs (Clarendon Press, New York, 1995).

20. A. WeIss, Rigidity of $p$-adic $p$-torsion, Ann. Math. (2) 127 (1988), 317-332.

21. A. WEIss, Torsion units in integral group rings, J. Reine Angew. Math. 415 (1991), 175-187.

22. H. Zassenhaus, On the torsion units of finite group rings, in Studies in mathematics (in honor of A. Almeida Costa), pp. 119-126 (Instituto de Alta Cultura, Lisbon, 1974; in Portuguese). 